\title{
Synthesis of Macrocycles Containing Endocyclic Chiral BINAM Moieties
}

\author{
Olga K. Grigorova, Alexei D. Averin, ${ }^{@}$ Olga A. Maloshitskaya, \\ and Irina P. Beletskaya \\ Lomonosov Moscow State University, Department of Chemistry, 119991 Moscow, Russia \\ ${ }^{\circledR}$ Corresponding author E-mail: alexaveron@yandex.ru
}

\begin{abstract}
A series of nitrogen- and oxygen-containing macrocycles with endocyclic chiral BINAM moieties were synthesized using Pd(0)-catalyzed amination with a number of oxadiamines. Two alternative ways to such compounds were compared - from 1,1'-binaphthalene-2,2'-diamine (BINAM) and N,N'-bis(3-bromophenyl) substituted oxadiamine and from $N, N$ '-bis(bromophenyl) substituted 1,1'-binaphthalene-2,2'-diamine and free oxadiamine. Macrocycles containing benzyl and 2,7-disubstituted naphthalene fragments were also successfully synthesized via the second approach, the dependence of their yields on the nature of starting compounds was demonstrated.
\end{abstract}

Keywords: Macrocycles, chirality, Pd catalysis, amination, polyamines.

\section{Синтез макроциклов, содержащих эндоциклические хиральные BINAM фрагменты}

\author{
О. К. Григорова, А. А. Аверин, ${ }^{@}$ О. А. Малошицкая, И. П. Белецкая
}

Московский государственный университет им. М.В. Ломоносова, 119991 Москва, Россия

${ }^{\circledR}$ E-mail: alexaveron@yandex.ru

\begin{abstract}
Синтезирован ряд азот- и кислородсодержащих макрочиклов с эндочиклическим хиральным фрагментом БИНАМ посредством Рd(0)-катализируемого аминирования с использованием ряда оксадиаминов. Произведено сравнение двух альтернативных путей получения таких соединений - из 1,1'-бинафталин-

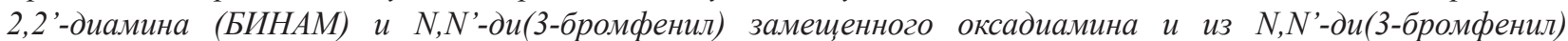
замещенного 1,1 '-бинафталин-2,2 '-диамина и свободного оксадиамина. Макроцикль, содержащие бензильный и 2,7-дизамещенный нафтильный фрагменты также успешно синтезированы с использованием второго метода, показана зависимость их выходов от природы исходных соединений.
\end{abstract}

Ключевые слова: Макроциклы, Pd катализ, хиральность, аминирование, полиамины.

\section{Introduction}

Macrocyclic compounds comprising endocyclic chiral moieties attract attention of researchers as biologically active compounds, perspective chemosensors for small chiral molecules, and ligands which can be used in enantioselective metal-catalyzed transformations. Such compounds occur in nature, among them the prominent place is occupied by macrolides, cyclic oligopeptides and cyclic oligosaccharides. These compounds contain multiple carbon chiral centers, and the majority of optically active synthetic macrocycles possess the same type of chirality. Enough rare are synthetic macrocyclic compounds with another type of chirality, i.e. planar-chiral macrocycles, ${ }^{[1-3]}$ and our previous works contributed to this field. ${ }^{[4,5]}$ Also we elaborated a new type of macrocycles with endocyclic chiral cholane fragments. ${ }^{[6-9]}$ Macrocyclic compounds containing fragments with $C 2$ chirality are known for quite a long time, and the majority 
of them possess 2,2'-binaphthol (BINOL) moiety. Some of them were reported before 1991 when a comprehensive review on all types of known macrocycles emerged. ${ }^{[10]}$ As this chiral moiety possesses fluorescent properties, BINOLbased chemosensors were found to be useful for detecting optically active amino alcohols, diamines, amino acids, $\alpha$-hydroxyacids. ${ }^{[11,12]}$ Reported are several macrocycles containing BINOL fragment which contain also additional chiral 1,2-diamine moieties. ${ }^{[13,14]}$ These compounds were synthesized via non-catalytic approaches, and in the course of our studies of the application of the catalytic amination to the synthesis of various nitrogen-containing macrocycles we decided to elaborate a general route to the macrocyclic species containing a parent chiral 1,1'-binaphthalene-2,2'diamine (BINAM) structural units. BINAM itself possesses interesting spectral properties, and in the combination with other aromatic and heteroaromatic groups the resulting $N$ and $O$-containing receptors could be perspective as optical chemosensors for small chiral organic molecules.

\section{Experimental}

NMR spectra were registered using Bruker Avance 400 spectrometer in $\mathrm{CDCl}_{3}$ at $298 \mathrm{~K}$. MALDI-TOF spectra were obtained with Bruker Autoflex II spectrometer using 1,8,9-trihydroxyanthracene as matrix and PEGs as internal standards. Dioxa-, trioxa- and tetraoxadiamines 1a-e, $(S)$-BINAM, 1,3-dibromobenzene, 1-(bromomethyl)-3-bromobenzene, phosphine ligands BINAP, DavePhos and Xantphos, potassium carbonate, sodium tert-butoxide, were purchased from Aldrich and used without further purification, $\operatorname{Pd}(\mathrm{dba})_{2}$ was synthesized according to the method described, ${ }^{[15]}$ 2,7-dibromonaphthalene was obtained via known procedure. ${ }^{[16]}$ Dioxane was distilled over $\mathrm{NaOH}$ followed by the distillation over sodium under argon, acetonitrile was distilled over $\mathrm{CaH}_{2}$, dichloromethane and methanol were used freshly distilled. Compound $\mathbf{2}$ was synthesized according to a procedure described. ${ }^{[17]}$

$7,14,15,17,18,21,22,28$-Octahydro- $13 \mathrm{H}, 20 \mathrm{H}-8,12: 27,23$ dimethenodinaphtho [1,2-p:2', 1'-n] $[1,4,7,13,18,24]$ dioxatetraazacyclohexacosine (3a). A two-neck flask equipped with a magnetic stirrer and reflux condenser, flushed with dry argon, was charged with compound 2 (0.1 mmol, $46 \mathrm{mg}), \operatorname{Pd}(\mathrm{dba})_{2}(8 \mathrm{~mol} \%$, $4.5 \mathrm{mg}$ ), BINAP (9 mol\%, $5.5 \mathrm{mg})$, absolute dioxane $(5 \mathrm{ml}),(S)$ BINAM $(0.1 \mathrm{mmol}, 28.5 \mathrm{mg})$ was then added followed by sodium tert-butoxide $(0.3 \mathrm{mmol}, 29 \mathrm{mg})$. The reaction mixture was refluxed for $24 \mathrm{~h}$, after cooling it down to ambient temperature the solvent was filtered, the precipitate was washed with $\mathrm{CH}_{2} \mathrm{Cl}_{2}(5 \mathrm{ml})$, combined organic fractions were evaporated in vacuo, and the residue was chromatographed on silica gel using a sequence of eluents: $\mathrm{CH}_{2} \mathrm{Cl}_{2}, \mathrm{CH}_{2} \mathrm{Cl}_{2} / \mathrm{MeOH}(200: 1-3: 1)$. The target compound 3a was obtained with the eluent $\mathrm{CH}_{2} \mathrm{Cl}_{2} / \mathrm{MeOH}(100: 1)$ as a brown solid. Yield $20 \mathrm{mg}$ (34\%). In the case when DavePhos (9 mol\%, mg) was used instead of BINAP, the yield was $43 \%$. (MALDI-TOF) found: 581.2876. $\mathrm{C}_{38} \mathrm{H}_{37} \mathrm{~N}_{4} \mathrm{O}_{2}$ requires $581.2917[\mathrm{M}+\mathrm{H}]^{+}$. ${ }^{1} \mathrm{H}$ NMR $\delta_{\mathrm{H}}$ ppm: $3.12-3.25(4 \mathrm{H}, \mathrm{m}), 3.61(4 \mathrm{H}, \mathrm{s}), 3.64-3.70(4 \mathrm{H}, \mathrm{m}), 5.51$ $(2 \mathrm{H}, \mathrm{s}), 6.08(2 \mathrm{H}, \mathrm{s}), 6.19\left(2 \mathrm{H}, \mathrm{dd},{ }^{3} J=8.0 \mathrm{~Hz},{ }^{4} J=1.5 \mathrm{~Hz}\right), 6.35$ $\left(2 \mathrm{H}, \mathrm{dd},{ }^{3} \mathrm{~J}=7.8 \mathrm{~Hz},{ }^{4} \mathrm{~J}=1.4 \mathrm{~Hz}\right), 6.97\left(2 \mathrm{H}, \mathrm{t},{ }^{3} \mathrm{~J}=8.0 \mathrm{~Hz}\right), 7.10(2 \mathrm{H}$, d, $\left.{ }^{3} J=8.5 \mathrm{~Hz}\right), 7.20-7.24(2 \mathrm{H}, \mathrm{m}), 7.29-7.33(2 \mathrm{H}, \mathrm{m}), 7.68(2 \mathrm{H}, \mathrm{d}$, $\left.{ }^{3} J=9.0 \mathrm{~Hz}\right), 7.84\left(2 \mathrm{H}, \mathrm{d},{ }^{3} J=8.1 \mathrm{~Hz}\right), 7.86\left(2 \mathrm{H}, \mathrm{d},{ }^{3} J=9.0 \mathrm{~Hz}\right)$, two $\mathrm{NH}$ protons were not assigned. ${ }^{13} \mathrm{C}$ NMR $\delta_{\mathrm{c}}$ ppm: $43.8(2 \mathrm{C}), 69.1$ (2C), 70.0 (2C), 104.3 (2C), 106.1 (2C), 109.2 (2C), 117.9 (2C), 119.4 (2C), 123.5 (2C), 124.9 (2C), 126.8 (2C), 128.1 (2C), 129.2 (2C), 129.6 (2C), 130.0 (2C), 134.4 (2C), 140.0 (2C), 144.1 (2C), 149.1 (2C).

$N^{2}, N^{2}$-Bis(3-bromophenyl)-(S)-1,1'-binaphthyl-2,2'-diamine (4). A two-neck flask equipped with a magnetic stirrer and reflux condenser, flushed with dry argon, was charged with 1,3-dibromo- benzene $(0.22 \mathrm{mmol}, 52 \mathrm{mg}), \mathrm{Pd}(\mathrm{dba})_{2}(2 \mathrm{~mol} \%, 1.2 \mathrm{mg})$, Xantphos $(2.5 \mathrm{~mol} \%, 1.2 \mathrm{mg})$, absolute dioxane $(1 \mathrm{ml}),(S)$-BINAM $(0.1 \mathrm{mmol}, 28.5 \mathrm{mg})$ was then added followed by sodium tert-butoxide $(0.3 \mathrm{mmol}, 29 \mathrm{mg})$. The reaction mixture was refluxed for $8 \mathrm{~h}$, after cooling it down to ambient temperature the solvent was filtered, the precipitate was washed with $\mathrm{CH}_{2} \mathrm{Cl}_{2}(5 \mathrm{ml})$, combined organic fractions were evaporated in vacuo, and the residue was chromatographed on silica gel using a sequence of eluents: hexanes/ $\mathrm{CH}_{2} \mathrm{Cl}_{2}(10: 1-1: 2)$. The target compound 4 was obtained with the eluent hexanes $/ \mathrm{CH}_{2} \mathrm{Cl}_{2}(10: 1)$ as a light-beige solid. Yield $47 \mathrm{mg}$ (68\%). (MALDI-TOF) found: 593.0251. $\mathrm{C}_{32} \mathrm{H}_{23} \mathrm{Br}_{2} \mathrm{~N}_{2}$ requires $593.0228[\mathrm{M}+\mathrm{H}]^{+} .{ }^{1} \mathrm{H}$ NMR $\delta_{\mathrm{H}}$ ppm: $5.46(2 \mathrm{H}$, br.s $), 6.72-6.77$ $(2 \mathrm{H}, \mathrm{m}), 6.94-6.98(6 \mathrm{H}, \mathrm{m}), 7.12\left(2 \mathrm{H}, \mathrm{d},{ }^{3} \mathrm{~J}=8.3 \mathrm{~Hz}\right), 7.23-7.27$ $(2 \mathrm{H}, \mathrm{m}), 7.34-7.38(2 \mathrm{H}, \mathrm{m}), 7.65\left(2 \mathrm{H}, \mathrm{d},{ }^{3} \mathrm{~J}=8.8 \mathrm{~Hz}\right), 7.87(2 \mathrm{H}, \mathrm{d}$, $\left.{ }^{3} J=8.0 \mathrm{~Hz}\right), 7.92\left(2 \mathrm{H}, \mathrm{d},{ }^{3} J=8.8 \mathrm{~Hz}\right) \cdot{ }^{13} \mathrm{C}$ NMR $\delta_{\mathrm{c}} \mathrm{ppm}: 117.0(2 \mathrm{C})$, 118.3 (2C), 118.9 (2C), 121.0 (2C), 123.0 (2C), 124.2 (2C), 124.4 (2C), $124.6(2 \mathrm{C}), 127.3(2 \mathrm{C}), 128.3$ (2C), 129.7 (2C), 130.0 (2C), 130.4 (2C), 133.8 (2C), 139.0 (2C), 144.3 (2C).

Typical procedure for the synthesis of macrocycles 3a-e. A two-neck flask equipped with a magnetic stirrer and reflux condenser is flushed with dry argon, charged with compound $\mathbf{4}$, $\mathrm{Pd}(\mathrm{dba})_{2}$, DavePhos, absolute dioxane, corresponding oxadiamine is then added followed by sodium tert-butoxide. The reaction mixture is refluxed for $24 \mathrm{~h}$, after cooling it down to ambient temperature the solvent is filtered, the precipitate is washed with $\mathrm{CH}_{2} \mathrm{Cl}_{2}$ $(5 \mathrm{ml})$, combined organic fractions are evaporated in vacuo, and the residue is chromatographed on silica gel using a sequence of eluents: $\mathrm{CH}_{2} \mathrm{Cl}_{2}, \mathrm{CH}_{2} \mathrm{Cl}_{2} / \mathrm{MeOH}(200: 1-3: 1)$.

$7,13,14,15,16,18,19,20,21,23,24,25,26,32$-Tetradecahydro8,12:31,27-dimethenodinaphtho[1,2-n:2',1'-1][1,26,5,11,16,22] dioxatetraazacyclotriacontine $(3 \mathrm{~b})$. Obtained from compound 4 $(0.17 \mathrm{mmol}, 100 \mathrm{mg})$, dioxadiamine $\mathbf{2 b}(0.17 \mathrm{mmol}, 34 \mathrm{mg})$, in the presence of $\operatorname{Pd}(\mathrm{dba})_{2}(8 \mathrm{~mol} \%, 8 \mathrm{mg})$, DavePhos $(8 \mathrm{~mol} \%, 6 \mathrm{mg})$, and sodium tert-butoxide $(0.51 \mathrm{mmol}, 48 \mathrm{mg})$ in $5 \mathrm{ml}$ of dioxane. Eluent: $\mathrm{CH}_{2} \mathrm{Cl}_{2} / \mathrm{MeOH}$ (50:1). Yield $27 \mathrm{mg}$ (25\%). (MALDI-TOF) found: 637.3590. $\mathrm{C}_{42} \mathrm{H}_{45} \mathrm{~N}_{4} \mathrm{O}_{2}$ requires $637.3543[\mathrm{M}+\mathrm{H}]^{+}$. ${ }^{1} \mathrm{H}$ NMR $\delta_{\mathrm{H}}$ ppm: $1.63-1.67(4 \mathrm{H}, \mathrm{m}), 1.80\left(4 \mathrm{H}\right.$, quintet, $\left.{ }^{3} \mathrm{~J}=5.9 \mathrm{~Hz}\right), 3.07(4 \mathrm{H}$, $\left.\mathrm{q},{ }^{3} \mathrm{~J}=5.7 \mathrm{~Hz}\right), 3.42-3.46(4 \mathrm{H}, \mathrm{m}), 3.50\left(4 \mathrm{H}, \mathrm{t},{ }^{3} \mathrm{~J}=5.6 \mathrm{~Hz}\right), 3.94(2 \mathrm{H}$, br.s), $5.51(2 \mathrm{H}, \mathrm{s}), 6.03\left(2 \mathrm{H}, \mathrm{t},{ }^{4} J=2.0 \mathrm{~Hz}\right), 6.20\left(2 \mathrm{H}, \mathrm{dd},{ }^{3} J=8.1 \mathrm{~Hz}\right.$, $\left.{ }^{4} J=2.0 \mathrm{~Hz}\right), 6.39\left(2 \mathrm{H}, \mathrm{dd},{ }^{3} J=7.8 \mathrm{~Hz},{ }^{4} J=2.0 \mathrm{~Hz}\right), 6.98(2 \mathrm{H}, \mathrm{t}$, $\left.{ }^{3} \mathrm{~J}=8.0 \mathrm{~Hz}\right), 7.12\left(2 \mathrm{H}, \mathrm{d},{ }^{3} \mathrm{~J}=8.3 \mathrm{~Hz}\right), 7.20-7.24(2 \mathrm{H}, \mathrm{m}), 7.28-7.32$ $(2 \mathrm{H}, \mathrm{m}), 7.69\left(2 \mathrm{H}, \mathrm{d},{ }^{3} \mathrm{~J}=9.0 \mathrm{~Hz}\right), 7.83\left(2 \mathrm{H}, \mathrm{d},{ }^{3} \mathrm{~J}=8.2 \mathrm{~Hz}\right), 7.86(2 \mathrm{H}$, d, $\left.{ }^{3} J=9.0 \mathrm{~Hz}\right) .{ }^{13} \mathrm{C}$ NMR $\delta_{c}$ ppm: $26.5(2 \mathrm{C}), 29.3(2 \mathrm{C}), 42.2(2 \mathrm{C})$, 69.3 (2C), 70.8 (2C), 104.2 (2C), 107.2 (2C), 108.8 (2C), 116.8 (2C), 118.9 (2C), 123.3 (2C), 124.7 (2C), 126.8 (2C), 128.1 (2C), 129.1 (2C), 129.4 (2C), 129.6 (2C), 134.1 (2C), 140.6 (2C), 143.9 (2C), $149.8(2 \mathrm{C})$.

$7,14,15,17,18,20,21,24,25,31$-Decahydro- $13 \mathrm{H}, 23 \mathrm{H}$ 8,12:30,26-dimethenodinaphtho[1,2-s:2', 1'-q][1,4,7,10,16,21,27] trioxatetraazacyclononacosine $(\mathbf{3 c})$. Obtained from compound 4 $(0.2 \mathrm{mmol}, 181 \mathrm{mg})$, trioxadiamine $2 \mathrm{c}(0.2 \mathrm{mmol}, 38 \mathrm{mg})$, in the presence of $\mathrm{Pd}(\mathrm{dba})_{2}$ (16 mol\%, $18 \mathrm{mg}$ ), DavePhos (18 mol\%, $14 \mathrm{mg}$ ), and sodium tert-butoxide $(0.6 \mathrm{mmol}, 58 \mathrm{mg})$ in $5 \mathrm{ml}$ of dioxane. Eluent: $\mathrm{CH}_{2} \mathrm{Cl}_{2} / \mathrm{MeOH}(100: 1)$. Yield $27 \mathrm{mg}$ (22\%). (MALDITOF) found: $625.3258 . \mathrm{C}_{40} \mathrm{H}_{41} \mathrm{~N}_{4} \mathrm{O}_{3}$ requires $625.3179[\mathrm{M}+\mathrm{H}]^{+}$. ${ }^{1} \mathrm{H}$ NMR $\delta_{\mathrm{H}}$ ppm: $3.04-3.16(4 \mathrm{H}, \mathrm{m}), 3.64(8 \mathrm{H}, \mathrm{s}), 3.65(4 \mathrm{H}, \mathrm{t}$, $\left.{ }^{3} J=5.1 \mathrm{~Hz}\right), 5.47(2 \mathrm{H}, \mathrm{s}), 6.05(2 \mathrm{H}, \mathrm{s}), 6.23\left(2 \mathrm{H}, \mathrm{d},{ }^{3} J=7.7 \mathrm{~Hz}\right), 6.34$ $\left(2 \mathrm{H}, \mathrm{d},{ }^{3} J=7.7 \mathrm{~Hz}\right), 6.93\left(2 \mathrm{H}, \mathrm{t},{ }^{3} J=8.0 \mathrm{~Hz}\right), 7.10\left(2 \mathrm{H}, \mathrm{d},{ }^{3} J=8.5 \mathrm{~Hz}\right)$, $7.20-7.24(2 \mathrm{H}, \mathrm{m}), 7.29-7.33(2 \mathrm{H}, \mathrm{m}), 7.65\left(2 \mathrm{H}, \mathrm{d},{ }^{3} J=9.0 \mathrm{~Hz}\right)$, $7.84\left(2 \mathrm{H}, \mathrm{d},{ }^{3} J_{o b s}=6.8 \mathrm{~Hz}\right), 7.86\left(2 \mathrm{H}, \mathrm{d},{ }^{3} J_{o b s}=8.6 \mathrm{~Hz}\right)$, two NH protons were not assigned.

$1,7,8,9,10,12,13,15,16,18,19,20,21,27$-Tetradecahydro2,6:26,22-dimethenodinaphtho[1,2-t:2',1'-r][1,4,7,11,17,22,28] trioxatetraazacyclohentriacontine $(\mathbf{3} \boldsymbol{d})$. Obtained from compound $4(0.13 \mathrm{mmol}, 77 \mathrm{mg})$, trioxadiamine $2 \mathbf{d}(0.13 \mathrm{mmol}, 29 \mathrm{mg})$, in the presence of Pd(dba) ${ }_{2}(8 \mathrm{~mol} \%, 6 \mathrm{mg})$, DavePhos ( $\left.9 \mathrm{~mol} \%, 4.5 \mathrm{mg}\right)$, and sodium tert-butoxide $(0.39 \mathrm{mmol}, 38 \mathrm{mg})$ in $5 \mathrm{ml}$ of dioxane. Eluent: $\mathrm{CH}_{2} \mathrm{Cl}_{2} / \mathrm{MeOH}(100: 1)$. Yield $22 \mathrm{mg}$ (25\%). (MALDI-TOF) 
found: $653.3528 . \mathrm{C}_{42} \mathrm{H}_{45} \mathrm{~N}_{4} \mathrm{O}_{3}$ requires $653.3492[\mathrm{M}+\mathrm{H}]^{+}$. ${ }^{1} \mathrm{H} \mathrm{NMR}$ $\delta_{\mathrm{H}}$ ppm: $1.78\left(4 \mathrm{H}\right.$, quintet, $\left.{ }^{3} \mathrm{~J}=6.0 \mathrm{~Hz}\right), 3.06\left(4 \mathrm{H}, \mathrm{t},{ }^{3} \mathrm{~J}=6.3 \mathrm{~Hz}\right), 3.55$ $\left(4 \mathrm{H}, \mathrm{t},{ }^{3} \mathrm{~J}=5.7 \mathrm{~Hz}\right), 3.57-3.60(4 \mathrm{H}, \mathrm{m}), 3.61-3.64(4 \mathrm{H}, \mathrm{m}), 3.70$ (2H, br.s), $5.46(2 \mathrm{H}, \mathrm{s}), 5.98(2 \mathrm{H}, \mathrm{s}), 6.22\left(2 \mathrm{H}, \mathrm{d},{ }^{3} \mathrm{~J}=7.8 \mathrm{~Hz}\right), 6.33$ $\left(2 \mathrm{H}, \mathrm{d},{ }^{3} J=7.8 \mathrm{~Hz}\right), 6.94\left(2 \mathrm{H}, \mathrm{t},{ }^{3} J=7.8 \mathrm{~Hz}\right), 7.12\left(2 \mathrm{H}, \mathrm{d},{ }^{3} J=8.5 \mathrm{~Hz}\right)$, $7.21-7.25(2 \mathrm{H}, \mathrm{m}), 7.29-7.33(2 \mathrm{H}, \mathrm{m}), 7.67\left(2 \mathrm{H}, \mathrm{d},{ }^{3} \mathrm{~J}=9.0 \mathrm{~Hz}\right)$, $7.84\left(2 \mathrm{H}, \mathrm{d},{ }^{3} J_{o b s}=6.4 \mathrm{~Hz}\right), 7.86\left(2 \mathrm{H}, \mathrm{d},{ }^{3} J_{o b s}=8.7 \mathrm{~Hz}\right) .{ }^{13} \mathrm{C} \mathrm{NMR} \delta_{c}$ ppm: 28.8 (2C), 42.0 (2C), 69.5 (2C), 70.2 (2C), $70.6(2 \mathrm{C}), 103.9$ (2C), 107.5 (2C), 108.8 (2C), 117.5 (2C), 119.5 (2C), 123.4 (2C), 124.8 (2C), 126.8 (2C), 128.1 (2C), 129.1 (2C), 129.5 (2C), 129.6 (2C), 134.2 (2C), 140.4 (2C), 144.2 (2C), 149.2 (2C).

$1,8,9,11,12,14,15,17,18,21,22,28$-Dodecahydro$7 H, 20 H-2,6: 27,23$-dimethenodinaphtho [1,2-v:2', 1'-t] $[1,4,7,10,13,19,24,30]$ tetraoxatetraazacyclodotriacontine (3e). Obtained from compound $4(0.1 \mathrm{mmol}, 59 \mathrm{mg})$, tetraoxadiamine $\mathbf{2 e}$ $(0.1 \mathrm{mmol}, 24 \mathrm{mg})$, in the presence of $\mathrm{Pd}(\mathrm{dba})_{2}(8 \mathrm{~mol} \%, 4.5 \mathrm{mg})$, DavePhos $(9 \mathrm{~mol} \%, 3 \mathrm{mg})$, and sodium tert-butoxide $(0.3 \mathrm{mmol}$, $28.5 \mathrm{mg}$ ) in $5 \mathrm{ml}$ of dioxane. Eluent: $\mathrm{CH}_{2} \mathrm{Cl}_{2} / \mathrm{MeOH}$ (100:1). Yield $18 \mathrm{mg}(27 \%)$. (MALDI-TOF) found: 669.3493. $\mathrm{C}_{42} \mathrm{H}_{45} \mathrm{~N}_{4} \mathrm{O}_{4}$ requires $669.3441[\mathrm{M}+\mathrm{H}]^{+} .{ }^{1} \mathrm{H}$ NMR $\delta_{\mathrm{H}}$ ppm: $3.05-3.12(4 \mathrm{H}, \mathrm{m})$, $3.58-3.66(16 \mathrm{H}, \mathrm{m}), 5.49(2 \mathrm{H}, \mathrm{s}), 6.09(2 \mathrm{H}$, br.s $), 6.28(2 \mathrm{H}$, d, $\left.{ }^{3} J=8.1 \mathrm{~Hz}\right), 6.35\left(2 \mathrm{H}, \mathrm{dd},{ }^{3} J=7.6 \mathrm{~Hz},{ }^{4} J=1.0 \mathrm{~Hz}\right), 6.93(2 \mathrm{H}, \mathrm{t}$, $\left.{ }^{3} J=8.0 \mathrm{~Hz}\right), 7.10\left(2 \mathrm{H}, \mathrm{d},{ }^{3} J=8.7 \mathrm{~Hz}\right), 7.21-7.25(2 \mathrm{H}, \mathrm{m}), 7.29-7.33$ $(2 \mathrm{H}, \mathrm{m}), 7.65\left(2 \mathrm{H}, \mathrm{d},{ }^{3} \mathrm{~J}=9.0 \mathrm{~Hz}\right), 7.84\left(2 \mathrm{H},{ }^{3} \mathrm{~J}_{o b s}=7.2 \mathrm{~Hz}\right), 7.86(2 \mathrm{H}$, ${ }^{3} J_{o b s}=8.7 \mathrm{~Hz}$ ), two $\mathrm{NH}$ protons were not assigned. ${ }^{13} \mathrm{C}$ NMR $\delta_{\mathrm{c}} \mathrm{ppm}$ : 44.0 (2C), 69.2 (2C), $70.3(2 \mathrm{C}), 70.6(2 \mathrm{C}), 70.7$ (2C), $104.2(2 \mathrm{C})$, 108.1 (2C), 109.5 (2C), 117.3 (2C), 119.2 (2C), 123.4 (2C), 124.8 (2C), 126.8 (2C), 128.1 (2C), 129.1 (2C), 129.5 (2C), 129.8 (2C), 134.2 (2C), 140.3 (2C), 144.1 (2C), 148.8 (2C).

$N^{2}, N^{2}$-Bis(7-bromonaphthalen-2-yl)-(S)-1,1'-binaphthyl-2,2'diamine (5). A two-neck flask equipped with a magnetic stirrer and reflux condenser, flushed with dry argon, was charged with 2,7-dibromonaphthalene (1.4 mmol, $400 \mathrm{mg}), \operatorname{Pd}(\mathrm{dba})_{2}$ (4 mol\%, $12 \mathrm{mg}$ ), Xantphos (4.5 mol\%, $13 \mathrm{mg})$, absolute dioxane (5 ml), (S)-BINAM $(0.5 \mathrm{mmol}, 142 \mathrm{mg})$ was then added followed by sodium tert-butoxide $(2 \mathrm{mmol}, 192 \mathrm{mg})$. The reaction mixture was refluxed for $8 \mathrm{~h}$, after cooling it down to ambient temperature the solvent was filtered, the precipitate was washed with $\mathrm{CH}_{2} \mathrm{Cl}_{2}(5 \mathrm{ml})$, combined organic fractions were evaporated in vacuo, and the residue was chromatographed on silica gel using a sequence of eluents: hexane $/ \mathrm{CH}_{2} \mathrm{Cl}_{2}$ $(10: 1-1: 2)$. The target compound 5 was obtained with the eluent hexanes $/ \mathrm{CH}_{2} \mathrm{Cl}_{2}$ (4:1) as a beige solid. Yield $180 \mathrm{mg}(52 \%)$. (MALDI-TOF) found: 693.0511. $\mathrm{C}_{40} \mathrm{H}_{27} \mathrm{Br}_{2} \mathrm{~N}_{2}$ requires 693.0541 [M+H] . ${ }^{1} \mathrm{H}$ NMR $\delta_{\mathrm{H}}$ ppm: 5.65 (2H, br.s), $6.81\left(2 \mathrm{H}, \mathrm{d},{ }^{3} J=8.8 \mathrm{~Hz}\right), 6.91(2 \mathrm{H}$, s), 7.19-7.44 (14H, m), $7.76\left(2 \mathrm{H}, \mathrm{d},{ }^{3} \mathrm{~J}=8.7 \mathrm{~Hz}\right), 7.93-7.98(4 \mathrm{H}, \mathrm{m})$. ${ }^{13} \mathrm{C}$ NMR $\delta_{\mathrm{c}}$ ppm: 110.4 (2C), 119.2 (2C), 119.9 (2C), 120.0 (2C), 120.4 (2C), 124.3 (2C), 124.7 (2C), 126.5 (2C), 127.2 (2C), 127.3 (2C), 128.0 (2C), 128.3 (2C), 128.9 (4C), 129.5 (2C), 130.1 (2C), 134.0 (2C), 135.2 (2C), 139.0 (2C), 141.7 (2C).

Typical procedure for the synthesis of macrocycles $\mathbf{6} \boldsymbol{a}, \boldsymbol{b}, \boldsymbol{d}, \boldsymbol{e}$. A two-neck flask equipped with a magnetic stirrer and reflux condenser is flushed with dry argon, charged with compound $\mathbf{5}$, $\mathrm{Pd}(\mathrm{dba})$, DavePhos, absolute dioxane, corresponding oxadiamine is then added followed by sodium tert-butoxide. The reaction mixture is refluxed for $24 \mathrm{~h}$, after cooling it down to ambient temperature the solvent is filtered, the precipitate is washed with $\mathrm{CH}_{2} \mathrm{Cl}_{2}(5 \mathrm{ml})$, combined organic fractions are evaporated in vacuo, and the residue is chromatographed on silica gel using a sequence of eluents: $\mathrm{CH}_{2} \mathrm{Cl}_{2}, \mathrm{CH}_{2} \mathrm{Cl}_{2} / \mathrm{MeOH}(200: 1-3: 1)$.

Macrocycle 6a. Obtained from compound $5(0.17 \mathrm{mmol}$, $117 \mathrm{mg})$, dioxadiamine 2a $(0.17 \mathrm{mmol}, 25 \mathrm{mg})$, in the presence of Pd(dba) $)_{2}(8 \mathrm{~mol} \%, 8 \mathrm{mg})$, DavePhos (9 mol\%, $6 \mathrm{mg}$ ), and sodium tert-butoxide $(0.51 \mathrm{mmol}, 49 \mathrm{mg})$ in $8 \mathrm{ml}$ dioxane. Eluent: $\mathrm{CH}_{2} \mathrm{Cl}_{2} / \mathrm{MeOH}$ (200:1). Yield $40 \mathrm{mg}$ (34\%). (MALDI-TOF) found: $681.3204 . \mathrm{C}_{46} \mathrm{H}_{41} \mathrm{~N}_{4} \mathrm{O}_{2}$ requires $681.3230[\mathrm{M}+\mathrm{H}]^{+}$. ${ }^{1} \mathrm{H}$ NMR $\delta_{\mathrm{H}}$ ppm: $3.26-3.33(4 \mathrm{H}, \mathrm{m}), 3.69(4 \mathrm{H}, \mathrm{s}), 3.78\left(4 \mathrm{H}, \mathrm{t},{ }^{3} J=4.7 \mathrm{~Hz}\right)$, $3.98(2 \mathrm{H}$, br.s $), 5.64(2 \mathrm{H}, \mathrm{s}), 6.30(2 \mathrm{H}, \mathrm{s}), 6.66(2 \mathrm{H}, \mathrm{s}), 6.68-6.76$ $(4 \mathrm{H}, \mathrm{m}), 7.18\left(2 \mathrm{H}, \mathrm{d},{ }^{3} \mathrm{~J}=8.5 \mathrm{~Hz}\right), 7.25-7.30(4 \mathrm{H}, \mathrm{m}), 7.33(2 \mathrm{H}$, d, $\left.{ }^{3} J=8.5 \mathrm{~Hz}\right), 7.38\left(2 \mathrm{H}, \mathrm{t},{ }^{3} \mathrm{~J}=7.4 \mathrm{~Hz}\right), 7.74(2 \mathrm{H}, \mathrm{d}, 9.0 \mathrm{~Hz}), 7.90$ $\left(2 \mathrm{H}, \mathrm{d},{ }^{3} J=8.1 \mathrm{~Hz}\right), 7.94\left(2 \mathrm{H}, \mathrm{d},{ }^{3} \mathrm{~J}=9.0 \mathrm{~Hz}\right)$, two NH protons were not assigned. ${ }^{13} \mathrm{C}$ NMR $\delta_{c}$ ppm: $53.4(2 \mathrm{C}), 60.1$ br. (2C), $69.4(2 \mathrm{C})$, 109.9 br. (2C), 114.7 (2C), 115.7 (2C), 121.1 br. (4C), 124.1 (2C), 125.1 (2C), 126.9 (2C), 128.2 (2C), 128.6 (2C), 128.7 (2C), 129.3 (2C), 130.1 (2C), 134.3 (2C), 135.4 br. (2C), 139.3 br. (2C), 142.1 (2C), six quaternary carbon atoms were not assigned due to line broadening.

Macrocycle $\mathbf{6} \boldsymbol{b}$. Obtained from compound $5(0.16 \mathrm{mmol}$, $110 \mathrm{mg})$, dioxadiamine $\mathbf{2 b}(0.16 \mathrm{mmol}, 33 \mathrm{mg})$, in the presence of Pd(dba) $)_{2}(8 \mathrm{~mol} \%, 7.5 \mathrm{mg})$, DavePhos ( $\left.9 \mathrm{~mol} \%, 6 \mathrm{mg}\right)$, and sodium tert-butoxide $(0.48 \mathrm{mmol}, 46 \mathrm{mg})$ in $5 \mathrm{ml}$ of dioxane. Eluent: $\mathrm{CH}_{2} \mathrm{Cl}_{2} / \mathrm{MeOH}$ (100:1). Yield $37 \mathrm{mg}$ (31\%). (MALDI-TOF) found: 737.3937. $\mathrm{C}_{50} \mathrm{H}_{49} \mathrm{~N}_{4} \mathrm{O}_{2}$ requires $737.3856[\mathrm{M}+\mathrm{H}]^{+}$. ${ }^{1} \mathrm{H}$ NMR $\delta_{\mathrm{H}}$ ppm: $1.70-1.74(4 \mathrm{H}, \mathrm{m}), 1.90\left(4 \mathrm{H}\right.$, quintet, $\left.{ }^{3} \mathrm{~J}=6.0 \mathrm{~Hz}\right), 3.13-3.26$ (4H, m), 3.48-3.52 (4H, m), $3.58\left(4 \mathrm{H}, \mathrm{t},{ }^{3} J=5.5 \mathrm{~Hz}\right), 5.71(2 \mathrm{H}$, s), $6.31(2 \mathrm{H}$, br.s $), 6.70\left(2 \mathrm{H}, \mathrm{dd},{ }^{3} J=8.3 \mathrm{~Hz},{ }^{4} J=1.5 \mathrm{~Hz}\right), 6.78(2 \mathrm{H}$, d, $\left.{ }^{4} J=2.3 \mathrm{~Hz}\right), 6.83\left(2 \mathrm{H}, \mathrm{dd},{ }^{3} J=8.3 \mathrm{~Hz},{ }^{4} J=2.3 \mathrm{~Hz}\right), 7.17(2 \mathrm{H}, \mathrm{d}$, $\left.{ }^{3} J=8.0 \mathrm{~Hz}\right), 7.23-7.27(2 \mathrm{H}, \mathrm{m}), 7.32-7.36(2 \mathrm{H}, \mathrm{m}), 7.43(4 \mathrm{H}, \mathrm{d}$, $\left.{ }^{3} \mathrm{~J}=8.7 \mathrm{~Hz}\right), 7.72\left(2 \mathrm{H}, \mathrm{d},{ }^{3} \mathrm{~J}=9.0 \mathrm{~Hz}\right), 7.87\left(2 \mathrm{H}, \mathrm{d},{ }^{3} \mathrm{~J}=8.0 \mathrm{~Hz}\right), 7.91$ $\left(2 \mathrm{H}, \mathrm{d},{ }^{3} \mathrm{~J}=8.8 \mathrm{~Hz}\right)$, two $\mathrm{NH}$ protons were not assigned. ${ }^{13} \mathrm{C} \mathrm{NMR}$ $\delta_{\mathrm{c}}$ ppm: 26.5 (2C), 29.1 (2C), 42.8 br. (2C), 69.3 (2C), 70.8 (2C), 112.5 (2C), 116.2 (2C), 117.0 (2C), 117.9 (2C), 119.7 (2C), 123.6 (2C), 124.9 (2C), 126.9 (2C), 128.2 (2C), 128.4 (2C), $128.6(2 \mathrm{C})$, 129.3 (2C), 129.7 (4C), 134.2 (2C), 136.2 (2C), 140.3 (2C), 141.3 (2C), four quaternary carbon atoms were not assigned due to line broadening.

Macrocycle 6d. Obtained from compound $5(0.26 \mathrm{mmol}$, $180 \mathrm{mg})$, trioxadiamine $2 \mathrm{~d}(0.26 \mathrm{mmol}, 57 \mathrm{mg})$, in the presence of Pd(dba) $)_{2}(8 \mathrm{~mol} \%, 12 \mathrm{mg}$ ), DavePhos ( $9 \mathrm{~mol} \%, 9 \mathrm{mg})$, and sodium tert-butoxide $(0.78 \mathrm{mmol}, 75 \mathrm{mg})$ in $8 \mathrm{ml}$ of dioxane. Eluent: $\mathrm{CH}_{2} \mathrm{Cl}_{2} / \mathrm{MeOH}$ (100:1). Yield $29 \mathrm{mg}$ (15\%). (MALDI-TOF) found: 753.3846. $\mathrm{C}_{50} \mathrm{H}_{49} \mathrm{~N}_{4} \mathrm{O}_{3}$ requires $753.3805[\mathrm{M}+\mathrm{H}]^{+} .{ }^{1} \mathrm{H}$ NMR $\delta_{\mathrm{H}} \mathrm{ppm}$ : $1.81-1.95(4 \mathrm{H}, \mathrm{m}), 3.12-3.25(4 \mathrm{H}, \mathrm{m}), 3.62-3.72(12 \mathrm{H}, \mathrm{m}), 5.68$ $(2 \mathrm{H}, \mathrm{s}), 6.15(2 \mathrm{H}, \mathrm{s}), 6.68(2 \mathrm{H}, \mathrm{s}), 6.71\left(2 \mathrm{H}, \mathrm{d},{ }^{3} \mathrm{~J}=9.0 \mathrm{~Hz}\right), 6.77$ $\left(2 \mathrm{H}, \mathrm{dd},{ }^{3} \mathrm{~J}=8.6 \mathrm{~Hz},{ }^{4} \mathrm{~J}=1.9 \mathrm{~Hz}\right), 7.18\left(2 \mathrm{H}, \mathrm{d},{ }^{3} \mathrm{~J}=8.3 \mathrm{~Hz}\right), 7.24-7.28$ $(2 \mathrm{H}, \mathrm{m}), 7.32-7.36(2 \mathrm{H}, \mathrm{m}), 7.38\left(2 \mathrm{H}, \mathrm{d},{ }^{3} J=8.7 \mathrm{~Hz}\right), 7.39(2 \mathrm{H}, \mathrm{d}$, $\left.{ }^{3} J=8.9 \mathrm{~Hz}\right), 7.71\left(2 \mathrm{H}, \mathrm{d},{ }^{3} J=8.8 \mathrm{~Hz}\right), 7.89\left(2 \mathrm{H}, \mathrm{d},{ }^{3} J=8.1 \mathrm{~Hz}\right), 7.91$ $\left(2 \mathrm{H}, \mathrm{d},{ }^{3} \mathrm{~J}=9.1 \mathrm{~Hz}\right)$, two $\mathrm{NH}$ protons were not assigned. ${ }^{13} \mathrm{C}$ NMR $\delta_{\mathrm{c}}$ ppm: $28.3(2 \mathrm{C}), 43.2$ br. (2C), $69.7(2 \mathrm{C}), 70.2(2 \mathrm{C}), 70.6(2 \mathrm{C})$, 115.6 (2C), 116.3 (2C), 116.8 (2C), 120.1 (2C), 123.8 (2C), 125.0 (2C), 126.9 (2C), $128.2(2 \mathrm{C}), 128.4(2 \mathrm{C}), 128.6(2 \mathrm{C}), 129.3(2 \mathrm{C})$, 129.7 (2C), 129.9 (2C), 134.3 (2C), 136.0 (2C), 140.0 (2C), 141.7 (2C), six quaternary carbon atoms were not assigned due to line broadening.

Macrocycle $\boldsymbol{6} \boldsymbol{e}$. Obtained from compound $5(0.1 \mathrm{mmol}$, $69 \mathrm{mg})$, tetraoxadiamine $2 \mathrm{e}(0.1 \mathrm{mmol}, 24 \mathrm{mg})$, in the presence of $\operatorname{Pd}(\mathrm{dba})_{2}(16 \mathrm{~mol} \%, 9 \mathrm{mg})$, DavePhos (18 mol\%, $7 \mathrm{mg}$ ), and sodium tert-butoxide $(0.3 \mathrm{mmol}, 29 \mathrm{mg})$ in $5 \mathrm{ml}$ of dioxane. Eluent: $\mathrm{CH}_{2} \mathrm{Cl}_{2} / \mathrm{MeOH}$ (100:1). Yield $5 \mathrm{mg}$ (7\%). (MALDI-TOF) found: 769.3815. $\mathrm{C}_{50} \mathrm{H}_{49} \mathrm{~N}_{4} \mathrm{O}_{4}$ requires $769.3754[\mathrm{M}+\mathrm{H}]^{+}$. ${ }^{1} \mathrm{H}$ NMR $\delta_{\mathrm{H}}$ ppm: 3.18 (4H, br.s), 3.65-3.75 (16H, m), $5.65(2 \mathrm{H}, \mathrm{s}), 6.22(2 \mathrm{H}$, br.s), $6.71(2 \mathrm{H}, \mathrm{s}), 6.73-6.79(4 \mathrm{H}, \mathrm{m}), 7.17\left(2 \mathrm{H}, \mathrm{d},{ }^{3} \mathrm{~J}=8.0 \mathrm{~Hz}\right), 7.28$ $\left(2 \mathrm{H}, \mathrm{d},{ }^{3} \mathrm{~J}=8.1 \mathrm{~Hz}\right), 7.31-7.40(6 \mathrm{H}, \mathrm{m}), 7.71\left(2 \mathrm{H}, \mathrm{d},{ }^{3} \mathrm{~J}=9.0 \mathrm{~Hz}\right)$, $7.89\left(2 \mathrm{H}, \mathrm{d},{ }^{3} J=8.6 \mathrm{~Hz}\right), 7.93\left(2 \mathrm{H}, \mathrm{d},{ }^{3} J=9.2 \mathrm{~Hz}\right)$, two $\mathrm{NH}$ protons were not assigned.

$N^{2}, N^{2}$-Bis(3-bromobenzyl)-(S)-1,1'-binaphthyl-2,2'-diamine (7). A two-neck flask equipped with a magnetic stirrer and reflux condenser, flushed with dry argon, was charged with (S)-BINAM (1 mmol, $284 \mathrm{mg})$, 1-(bromomethyl)-3-bromobenzene (2 mmol, $496 \mathrm{mg})$, acetonitrile $(10 \mathrm{ml})$ and potassium carbonate $(4 \mathrm{mmol}$, $552 \mathrm{mg}$ ). The reaction mixture was stirred at reflux for $24 \mathrm{~h}$, after cooling it down to ambient temperature the solvent was filtered, the precipitate was washed with $\mathrm{CH}_{2} \mathrm{Cl}_{2}(5 \mathrm{ml})$, combined organic fractions were evaporated in vacuo, and the residue was chromatographed on silica gel using a sequence of eluents: hexanes $/ \mathrm{CH}_{2} \mathrm{Cl}_{2}$ ( $4: 1-1: 2), \mathrm{CH}_{2} \mathrm{Cl}_{2}$. The target compound 7 was obtained with the eluent hexanes/ $\mathrm{CH}_{2} \mathrm{Cl}_{2}$ (1:1) as a yellow viscous oil. Yield $199 \mathrm{mg}$ 
(32\%). (MALDI-TOF) found: 621.0505. $\mathrm{C}_{34} \mathrm{H}_{27} \mathrm{Br}_{2} \mathrm{~N}_{2}$ requires $621.0541[\mathrm{M}+\mathrm{H}]^{+} .{ }^{1} \mathrm{H}$ NMR $\delta_{\mathrm{H}}$ ppm: 4.35 (2H, br.s), $4.40(4 \mathrm{H}, \mathrm{s})$, $7.08-7.16(8 \mathrm{H}, \mathrm{m}), 7.24-7.30(4 \mathrm{H}, \mathrm{m}), 7.33\left(2 \mathrm{H}, \mathrm{d},{ }^{3} J=7.7 \mathrm{~Hz}\right)$, $7.40(2 \mathrm{H}, \mathrm{s}), 7.78-7.81(2 \mathrm{H}, \mathrm{m}), 7.84\left(2 \mathrm{H}, \mathrm{d},{ }^{3} \mathrm{~J}=9.0 \mathrm{~Hz}\right) .{ }^{13} \mathrm{C}$ NMR $\delta_{\mathrm{c}}$ ppm: 46.7 (2C), $112.0(2 \mathrm{C}), 113.9$ (2C), 122.3 (2C), $122.7(2 \mathrm{C})$, 123.7 (2C), 125.3 (2C), 127.1 (2C), 127.8 (2C), 128.2 (2C), 129.6 (2C), 129.8 (2C), 130.0 (2C), 133.7 (2C), 142.3 (2C), 143.5 (2C).

$N^{2}, N^{2}, N^{2}$-Tris(3-bromobenzyl)-(S)-1,1'-binaphthyl-2,2'diamine (9). Obtained as the second product in the synthesis of compound 7. Eluent: hexanes/ $\mathrm{CH}_{2} \mathrm{Cl}_{2}(2: 1)$. Yield $117 \mathrm{mg}(15 \%)$. (MALDI-TOF) found: 789.0180. $\mathrm{C}_{41} \mathrm{H}_{32} \mathrm{Br}_{3} \mathrm{~N}_{2}$ requires 789.0116 $[\mathrm{M}+\mathrm{H}]^{+}$. ${ }^{1} \mathrm{H}$ NMR $\delta_{\mathrm{H}}$ ppm: $3.98\left(2 \mathrm{H}, \mathrm{d},{ }^{2} J=14.7 \mathrm{~Hz}\right), 4.00(1 \mathrm{H}$, br.s), $4.03\left(2 \mathrm{H}, \mathrm{d},{ }^{2} J=14.7 \mathrm{~Hz}\right), 4.25\left(1 \mathrm{H}, \mathrm{d},{ }^{2} J=15.8 \mathrm{~Hz}\right), 4.42$ $\left(1 \mathrm{H}, \mathrm{d},{ }^{2} \mathrm{~J}=15.8 \mathrm{~Hz}\right), 6.77\left(2 \mathrm{H}, \mathrm{d},{ }^{3} J=7.6 \mathrm{~Hz}\right), 6.95-7.02(7 \mathrm{H}, \mathrm{m})$, $7.12\left(1 \mathrm{H}, \mathrm{d},{ }^{3} J=8.8 \mathrm{~Hz}\right), 7.18-7.28(5 \mathrm{H}, \mathrm{m}), 7.30-7.36(2 \mathrm{H}, \mathrm{m})$, $7.43\left(1 \mathrm{H}\right.$, ddd, $\left.{ }^{3} J=7.8 \mathrm{~Hz},{ }^{3} J=6.1 \mathrm{~Hz},{ }^{4} J=1.9 \mathrm{~Hz}\right), 7.48(1 \mathrm{H}, \mathrm{d}$, $\left.{ }^{3} J=8.9 \mathrm{~Hz}\right), 7.80-7.85(2 \mathrm{H}, \mathrm{m}), 7.92\left(1 \mathrm{H}, \mathrm{d},{ }^{3} J=8.1 \mathrm{~Hz}\right), 7.96(1 \mathrm{H}$, d, $\left.{ }^{3} J=8.8 \mathrm{~Hz}\right) \cdot{ }^{13} \mathrm{C}$ NMR $\delta_{\mathrm{c}} \mathrm{ppm}: 47.1$ (1C), $56.2(2 \mathrm{C}), 113.7$ (1C), 115.5 (1C), 122.1 (1C), 122.2 (2C), 122.6 (1C), 124.1 (1C), 124.9 (1C), 125.2 (1C), 125.3 (1C), 125.8 (1C), 126.7 (1C), 127.0 (1C), 127.1 (2C), 127.7 (1C), 128.1 (1C), 128.3 (1C), 129.1 (1C), 129.4 (3C), 129.7 (1C), 129.9 br. (4C), 130.8 (1C), 131.5 (2C), 133.6 (2C), 140.5 (3C), 142.2 (1C), 142.5 (1C), 149.0 (1C).

$N^{2}, N^{2}$-Bis(3-bromobenzyl)-(S)-1,1 '-binaphthyl-2,2'-diamine (10). Obtained as the third product in the synthesis of compound 7. Eluent: hexanes $/ \mathrm{CH}_{2} \mathrm{Cl}_{2}(1: 2)$. Yield $31 \mathrm{mg}(5 \%)$ (in the mixture with compound 7). (MALDI-TOF) found: 621.0488 . $\mathrm{C}_{34} \mathrm{H}_{27} \mathrm{Br}_{2} \mathrm{~N}_{2}$ requires $621.0541[\mathrm{M}+\mathrm{H}]^{+} .{ }^{1} \mathrm{H} \quad \mathrm{NMR} \delta_{\mathrm{H}}$ ppm: $3.98\left(2 \mathrm{H}, \mathrm{d},{ }^{2} J=14.8 \mathrm{~Hz}\right), 4.03\left(2 \mathrm{H}, \mathrm{d},{ }^{2} J=14.8 \mathrm{~Hz}\right), 6.81(2 \mathrm{H}, \mathrm{d}$, $\left.{ }^{3} J=7.7 \mathrm{~Hz}\right), 7.00\left(2 \mathrm{H}, \mathrm{t},{ }^{3} J=7.8 \mathrm{~Hz}\right), 7.03\left(1 \mathrm{H}, \mathrm{d},{ }^{3} J=7.8 \mathrm{~Hz}\right), 7.06$ $(2 \mathrm{H}, \mathrm{s}), 7.10-7.35(10 \mathrm{H}, \mathrm{m}), 7.24\left(1 \mathrm{H}, \mathrm{ddd},{ }^{3} \mathrm{~J}=8.3 \mathrm{~Hz},{ }^{3} \mathrm{~J}=6.8 \mathrm{~Hz}\right.$, $\left.{ }^{4} J=1.4 \mathrm{~Hz}\right), 7.42-7.46(1 \mathrm{H}, \mathrm{m}), 7.53\left(1 \mathrm{H}, \mathrm{d},{ }^{3} J=8.8 \mathrm{~Hz}\right), 7.83-7.89$ $(2 \mathrm{H}, \mathrm{m}), 7.93\left(1 \mathrm{H}, \mathrm{d},{ }^{3} J=8.2 \mathrm{~Hz}\right), 7.98\left(1 \mathrm{H}, \mathrm{d},{ }^{3} J=8.8 \mathrm{~Hz}\right)$, two $\mathrm{NH}$ protons were not assigned.

$N^{2}$-(3-Bromobenzyl)-(S)-1,1'-binaphthyl-2,2'-diamine (11). Obtained as the fourth product in the synthesis of compound 7. Eluent: $\mathrm{CH}_{2} \mathrm{Cl}_{2}$. Yield $100 \mathrm{mg}(22 \%)$. (MALDI-TOF) found: 453.0937. $\mathrm{C}_{27} \mathrm{H}_{22} \mathrm{BrN}_{2}$ requires $453.0966[\mathrm{M}+\mathrm{H}]^{+} .{ }^{1} \mathrm{H} \mathrm{NMR} \delta_{\mathrm{H}} \mathrm{ppm}: 3.83(3 \mathrm{H}$, br.s), $4.36(2 \mathrm{H}, \mathrm{s}), 7.07-7.15(5 \mathrm{H}, \mathrm{m}), 7.17\left(1 \mathrm{H}, \mathrm{d},{ }^{3} \mathrm{~J}=8.8 \mathrm{~Hz}\right)$, $7.19-7.23(2 \mathrm{H}, \mathrm{m}), 7.26-7.30(2 \mathrm{H}, \mathrm{m}), 7.32\left(1 \mathrm{H}, \mathrm{d},{ }^{3} J=7.7 \mathrm{~Hz}\right)$, $7.40(1 \mathrm{H}, \mathrm{s}), 7.77-7.85(4 \mathrm{H}, \mathrm{m}) .{ }^{13} \mathrm{C}$ NMR $\delta_{\mathrm{c}} \mathrm{ppm}: 46.8,112.0$, 112.6, 113.9, 118.3, 122.1, 122.5, 122.7, 123.7, 123.9, 125.4, 126.8, $127.0,127.7,128.1,128.2,128.5,129.6,129.7$ (2C), $130.0(2 \mathrm{C})$, $133.5,133.9,142.4,142.9,143.3$.

Typical procedure for the synthesis of macrocycles $\mathbf{1 2 a}, \boldsymbol{b}, \boldsymbol{d}$. A two-neck flask equipped with a magnetic stirrer and reflux condenser is flushed with dry argon, charged with compound 7, $\mathrm{Pd}(\mathrm{dba})_{2}$, BINAP, absolute dioxane, corresponding oxadiamine is then added followed by sodium tert-butoxide. The reaction mixture is refluxed for $24 \mathrm{~h}$, after cooling it down to ambient temperature the solvent is filtered, the precipitate is washed with $\mathrm{CH}_{2} \mathrm{Cl}_{2}(5 \mathrm{ml})$, combined organic fractions are evaporated in vacuo, and the residue is chromatographed on silica gel using a sequence of eluents: $\mathrm{CH}_{2} \mathrm{Cl}_{2}$, $\mathrm{CH}_{2} \mathrm{Cl}_{2} / \mathrm{MeOH}(200: 1-3: 1)$.

$7,8,15,16,18,19,22,23,29,30$-Decahydro- $14 \mathrm{H}, 21 \mathrm{H}$ 9,13:28,24-dimethenodinaphtho[1,2-q:2',1'-o][1,4,7,14,19,26] dioxatetraazacyclooctacosine (12a). Obtained from compound 7 $(0.13 \mathrm{mmol}, 82 \mathrm{mg})$, dioxadiamine $2 \mathrm{a}(0.13 \mathrm{mmol}, 19 \mathrm{mg})$, in the presence of $\mathrm{Pd}(\mathrm{dba})_{2}(8 \mathrm{~mol} \%, 6 \mathrm{mg})$, BINAP $(9 \mathrm{~mol} \%, 8 \mathrm{mg})$, and sodium tert-butoxide $(0.39 \mathrm{mmol}, 40 \mathrm{mg})$ in $4 \mathrm{ml}$ of dioxane. Eluent: $\mathrm{CH}_{2} \mathrm{Cl}_{2} / \mathrm{MeOH}$ (100:1). Yield $32 \mathrm{mg}$ (41\%). (MALDI-TOF) found: 609.3180. $\mathrm{C}_{40} \mathrm{H}_{41} \mathrm{~N}_{4} \mathrm{O}_{2}$ requires $609.3230[\mathrm{M}+\mathrm{H}]^{+} .{ }^{1} \mathrm{H} \mathrm{NMR}$ $\delta_{\mathrm{H}} \mathrm{ppm}: 3.11\left(4 \mathrm{H}, \mathrm{t},{ }^{3} J=4.9 \mathrm{~Hz}\right), 3.53\left(4 \mathrm{H}, \mathrm{t},{ }^{3} J=4.9 \mathrm{~Hz}\right), 3.54(4 \mathrm{H}$, s), $4.22\left(2 \mathrm{H}, \mathrm{d},{ }^{2} J=15.3 \mathrm{~Hz}\right), 4.34\left(2 \mathrm{H}, \mathrm{d},{ }^{2} J=15.3 \mathrm{~Hz}\right), 4.68(2 \mathrm{H}$, s), $6.47\left(2 \mathrm{H}, \mathrm{d},{ }^{3} J=8.5 \mathrm{~Hz}\right), 6.49(2 \mathrm{H}, \mathrm{s}), 6.56\left(2 \mathrm{H}, \mathrm{d},{ }^{3} J=7.2 \mathrm{~Hz}\right)$, $6.99-7.05(4 \mathrm{H}, \mathrm{m}), 7.14-7.22(4 \mathrm{H}, \mathrm{m}), 7.24\left(2 \mathrm{H}, \mathrm{d},{ }^{3} J=7.5 \mathrm{~Hz}\right)$, 7.73-7.76 (2H, m), $7.82\left(2 \mathrm{H}, \mathrm{d},{ }^{3} J=9.0 \mathrm{~Hz}\right)$, two $\mathrm{NH}$ protons were not assigned. ${ }^{13} \mathrm{C}$ NMR $\delta_{\mathrm{c}}$ ppm: 43.7 (2C), 48.3 (2C), 69.3 (2C),
70.2 (2C), $111.6(2 \mathrm{C}), 112.1(4 \mathrm{C}), 114.6(2 \mathrm{C}), 116.5(2 \mathrm{C}), 121.9$ (2C), 123.9 (2C), 126.7 (2C), 127.8 (2C), 128.1 (2C), 129.3 (2C), $129.6(2 \mathrm{C}), 133.8$ (2C), 141.0 (2C), 144.7 (2C), 148.1 (2C).

$1,2,8,9,10,11,13,14,15,16,18,19,20,21,27,28$-Hexadecahydro-3, 7:26,22-dimethenodinaphtho [1,2-o:2', 1'-m] $[1,28,5,12,17,24]$ dioxatetraazacyclodotriacontine (12b). Obtained from compound 7 (0.13 mmol, $82 \mathrm{mg})$, dioxadiamine $\mathbf{2 b}$ $(0.13 \mathrm{mmol}, 26 \mathrm{mg})$, in the presence of $\operatorname{Pd}(\mathrm{dba})_{2}(8 \mathrm{~mol} \%, 6 \mathrm{mg})$, BINAP $(9 \mathrm{~mol} \%, 8 \mathrm{mg})$, and sodium tert-butoxide $(0.39 \mathrm{mmol}$, $40 \mathrm{mg}$ ) in $4 \mathrm{ml}$ of dioxane. Eluent: $\mathrm{CH}_{2} \mathrm{Cl}_{2} / \mathrm{MeOH}$ (200:1). Yield $38 \mathrm{mg}$ (44\%). (MALDI-TOF) found: 665.3817. $\mathrm{C}_{44} \mathrm{H}_{49} \mathrm{~N}_{4} \mathrm{O}_{2}$ requires $665.3856[\mathrm{M}+\mathrm{H}]^{+} .{ }^{1} \mathrm{H}$ NMR $\delta_{\mathrm{H}} \mathrm{ppm}: 1.62-1.66(4 \mathrm{H}, \mathrm{m})$, $1.77\left(4 \mathrm{H}\right.$, quintet, $\left.{ }^{3} \mathrm{~J}=5.7 \mathrm{~Hz}\right), 3.09\left(4 \mathrm{H}, \mathrm{t},{ }^{3} \mathrm{~J}=6.0 \mathrm{~Hz}\right), 3.38-3.42$ $(4 \mathrm{H}, \mathrm{m}), 3.46\left(4 \mathrm{H}, \mathrm{t},{ }^{3} \mathrm{~J}=5.4 \mathrm{~Hz}\right), 4.19-4.38(6 \mathrm{H}, \mathrm{m}), 6.40(2 \mathrm{H}$, d, $\left.{ }^{3}=7.5 \mathrm{~Hz}\right), 6.46(2 \mathrm{H}, \mathrm{s}), 6.50\left(2 \mathrm{H}, \mathrm{d},{ }^{3} J=8.0 \mathrm{~Hz}\right), 6.98(2 \mathrm{H}, \mathrm{t}$, $\left.{ }^{3} J=7.7 \mathrm{~Hz}\right), 7.01-7.05(2 \mathrm{H}, \mathrm{m}), 7.15-7.21(6 \mathrm{H}, \mathrm{m}), 7.73-7.77(2 \mathrm{H}$, $\mathrm{m}), 7.80\left(2 \mathrm{H}, \mathrm{d},{ }^{3} \mathrm{~J}=8.9 \mathrm{~Hz}\right)$, two $\mathrm{NH}$ protons were not assigned. ${ }^{13} \mathrm{C}$ NMR $\delta_{c}$ ppm: 26.7 (2C), 29.2 (2C), 42.1 (2C), 47.7 (2C), 69.5 (2C), $70.8(2 \mathrm{C}), 110.8(2 \mathrm{C}), 111.1(2 \mathrm{C}), 111.9$ (2C), $114.3(2 \mathrm{C}), 115.4$ (2C), 121.8 (2C), 123.9 (2C), 126.6 (2C), 128.0 (2C), 129.3 (2C), 129.5 (2C), 133.9 (2C), 140.9 (2C), 144.4 (2C), 148.9 (2C).

$1,2,8,9,10,11,13,14,16,17,19,20,21,22,28,29$-Hexadecahydro3,7:27,23-dimethenodinaphtho[1,2-u:2',1'-s][1,4,7,11,18,23,30] trioxatetraazacyclotritriacontine (12d). Obtained from compound $7(0.13 \mathrm{mmol}, 82 \mathrm{mg})$, trioxadiamine $2 \mathrm{~d}(0.13 \mathrm{mmol}, 29 \mathrm{mg})$, in the presence of Pd(dba) $(8 \mathrm{~mol} \%, 6 \mathrm{mg})$, BINAP $(9 \mathrm{~mol} \%, 8 \mathrm{mg})$, and sodium tert-butoxide $(0.39 \mathrm{mmol}, 40 \mathrm{mg})$ in $4 \mathrm{ml}$ of dioxane. Eluent: $\mathrm{CH}_{2} \mathrm{Cl}_{2} / \mathrm{MeOH}$ (100:1). Yield $39 \mathrm{mg}$ (45\%). (MALDI-TOF) found: $681.3759 . \mathrm{C}_{44} \mathrm{H}_{49} \mathrm{~N}_{4} \mathrm{O}_{3}$ requires $681.3805[\mathrm{M}+\mathrm{H}]^{+} .{ }^{1} \mathrm{H} \mathrm{NMR}$ $\delta_{\mathrm{H}}$ ppm: $1.74\left(4 \mathrm{H}\right.$, quintet, $\left.{ }^{3} \mathrm{~J}=6.0 \mathrm{~Hz}\right), 3.08\left(4 \mathrm{H}, \mathrm{t},{ }^{3} \mathrm{~J}=6.3 \mathrm{~Hz}\right), 3.52$ $\left(4 \mathrm{H}, \mathrm{t},{ }^{3} \mathrm{~J}=5.8 \mathrm{~Hz}\right), 3.54-3.57(4 \mathrm{H}, \mathrm{m}), 3.60-3.63(4 \mathrm{H}, \mathrm{m}), 4.25$ ( $\left.2 \mathrm{H}, \mathrm{d},{ }^{2} J=15.7 \mathrm{~Hz}\right), 4.30$ (2H, br.s), $4.44\left(2 \mathrm{H}, \mathrm{d},{ }^{2} J=15.7 \mathrm{~Hz}\right), 6.48$ $\left(2 \mathrm{H}, \mathrm{d},{ }^{3} \mathrm{~J}=7.7 \mathrm{~Hz}\right), 6.52(2 \mathrm{H}, \mathrm{s}), 6.56\left(2 \mathrm{H}, \mathrm{d},{ }^{3} \mathrm{~J}=7.6 \mathrm{~Hz}\right), 6.99-7.04$ $(4 \mathrm{H}, \mathrm{m}), 7.14-7.20(6 \mathrm{H}, \mathrm{m}), 7.73-7.77(2 \mathrm{H}, \mathrm{m}), 7.81(2 \mathrm{H}, \mathrm{d}$, ${ }^{3} J=9.0 \mathrm{~Hz}$ ), two $\mathrm{NH}$ protons were not assigned. ${ }^{13} \mathrm{C}$ NMR $\delta_{\mathrm{c}} \mathrm{ppm}$ : $28.6(2 \mathrm{C}), 42.3$ (2C), 47.7 (2C), 69.8 (2C), 70.1 (2C), 70.6 (2C), $111.6(4 \mathrm{C}), 111.8(2 \mathrm{C}), 114.2(2 \mathrm{C}), 116.0(2 \mathrm{C}), 121.8(2 \mathrm{C}), 123.9$ (2C), 126.6 (2C), 127.7 (2C), 128.1 (2C), 129.3 (2C), 129.6 (2C), 133.9 (2C), 141.0 (2C), 144.4 (2C), 148.2 (2C).

$N^{2}, N^{2}$-Bis(3,5-dibromobenzyl)-(S)-1,1'-binaphthyl-2,2'-diamine (13). A two-neck flask equipped with a magnetic stirrer and reflux condenser, flushed with dry argon, was charged with $(S)$-BINAM (2.4 mmol, 674mg), 1,3-dibromo-5-(bromomethyl)benzene $(4.8 \mathrm{mmol}, 1551 \mathrm{mg})$, acetonitrile $(24 \mathrm{ml})$ and potassium carbonate $(9.6 \mathrm{mmol}, 1325 \mathrm{mg})$. The reaction mixture was stirred at reflux for $24 \mathrm{~h}$, after cooling it down to ambient temperature the solvent was filtered, the precipitate was washed with $\mathrm{CH}_{2} \mathrm{Cl}_{2}(10 \mathrm{ml})$, combined organic fractions were evaporated in vacuo, and the residue was chromatographed on silica gel using a sequence of eluents: hexanes $/ \mathrm{CH}_{2} \mathrm{Cl}_{2}(10: 1-1: 1)$. The target compound $\mathbf{1 3}$ was obtained with the eluent hexanes $/ \mathrm{CH}_{2} \mathrm{Cl}_{2}(2: 1)$ as a yellow viscous oil. Yield $1424 \mathrm{mg}\left(76 \%\right.$ ). (MALDI-TOF) found: 776.8662. $\mathrm{C}_{34} \mathrm{H}_{25} \mathrm{Br}_{4} \mathrm{~N}_{2}$ requires $776.8751[\mathrm{M}+\mathrm{H}]^{+} .{ }^{1} \mathrm{H}$ NMR $\delta_{\mathrm{H}} \mathrm{ppm}: 4.41(4 \mathrm{H}, \mathrm{s}), 4.45(2 \mathrm{H}$, br.s), $7.11\left(2 \mathrm{H}, \mathrm{d},{ }^{3} J=9.0 \mathrm{~Hz}\right), 7.21\left(2 \mathrm{H}, \mathrm{d},{ }^{3} J=8.3 \mathrm{~Hz}\right), 7.23(2 \mathrm{H}$, ddd, $\left.{ }^{3} J=8.1 \mathrm{~Hz},{ }^{3} J=7.0 \mathrm{~Hz},{ }^{4} J=1.3 \mathrm{~Hz}\right), 7.37\left(4 \mathrm{H}, \mathrm{d},{ }^{4} J=1.8 \mathrm{~Hz}\right), 7.38$ $\left(2 \mathrm{H}, \mathrm{ddd},{ }^{3} J=8.3 \mathrm{~Hz},{ }^{3} J=7.0 \mathrm{~Hz},{ }^{4} \mathrm{~J}=1.4 \mathrm{~Hz}\right), 7.55\left(2 \mathrm{H}, \mathrm{t},{ }^{4} \mathrm{~J}=1.8 \mathrm{~Hz}\right)$, $7.86\left(2 \mathrm{H}, \mathrm{d},{ }^{3} J=7.8 \mathrm{~Hz}\right), 7.90\left(2 \mathrm{H},{ }^{3} J=9.0 \mathrm{~Hz}\right) .{ }^{13} \mathrm{C}$ NMR $\delta_{\mathrm{c}} \mathrm{ppm}:$ 46.0 (2C), 111.9 (2C), 113.5 (2C), 122.5 (2C), 123.1 (4C), 123.6 (2C), 127.4 (2C), 127.8 (2C), 128.3 (6C), 130.0 (2C), 132.5 (2C), $133.6(2 \mathrm{C}), 142.9(2 \mathrm{C}), 144.1$ (2C).

$N^{2}, N^{2}, N^{2}$-Tris(3,5-dibromobenzyl)-(S)-1,1'-binaphthyl-2,2'diamine (14). Obtained as the second product in the synthesis of compound 13. Eluent: hexanes $/ \mathrm{CH}_{2} \mathrm{Cl}_{2}$ (10:1). Yield $150 \mathrm{mg}(9 \%)$. (MALDI-TOF) found: 1022.71. $\mathrm{C}_{41} \mathrm{H}_{29} \mathrm{Br}_{6} \mathrm{~N}_{2}$ requires 1022.74 $[\mathrm{M}+\mathrm{H}]^{+} .{ }^{1} \mathrm{H}$ NMR $\delta_{\mathrm{H}}$ ppm: 3.95 (1H, br.s), $3.97(4 \mathrm{H}, \mathrm{s}), 4.22(1 \mathrm{H}$, $\left.\mathrm{dd},{ }^{2} J=16.8 \mathrm{~Hz},{ }^{3} J=4.8 \mathrm{~Hz}\right), 4.43\left(1 \mathrm{H}, \mathrm{dd},{ }^{2} J=16.8 \mathrm{~Hz},{ }^{3} J=6.8 \mathrm{~Hz}\right)$, $6.81\left(4 \mathrm{H}, \mathrm{d},{ }^{4} \mathrm{~J}=1.5 \mathrm{~Hz}\right), 6.89-6.92(1 \mathrm{H}, \mathrm{m}), 7.06\left(1 \mathrm{H}, \mathrm{d},{ }^{3} \mathrm{~J}=9.0 \mathrm{~Hz}\right)$, $7.15\left(2 \mathrm{H}, \mathrm{d},{ }^{4} \mathrm{~J}=1.3 \mathrm{~Hz}\right), 7.19-7.27(2 \mathrm{H}, \mathrm{m}), 7.31\left(1 \mathrm{H}, \mathrm{d},{ }^{3} \mathrm{~J}=8.2 \mathrm{~Hz}\right)$, 
$7.37\left(2 \mathrm{H}, \mathrm{t},{ }^{4} \mathrm{~J}=1.5 \mathrm{~Hz}\right), 7.37-7.41(1 \mathrm{H}, \mathrm{m}), 7.43\left(1 \mathrm{H}, \mathrm{t},{ }^{3} \mathrm{~J}=1.3 \mathrm{~Hz}\right)$, $7.45-7.49(1 \mathrm{H}, \mathrm{m}), 7.53\left(1 \mathrm{H}, \mathrm{d},{ }^{3} \mathrm{~J}=9.0 \mathrm{~Hz}\right), 7.80-7.83(1 \mathrm{H}, \mathrm{m}), 7.86$ $\left(1 \mathrm{H}, \mathrm{d},{ }^{3} \mathrm{~J}=9.1 \mathrm{~Hz}\right), 7.94\left(1 \mathrm{H},{ }^{3} \mathrm{~J}=8.1 \mathrm{~Hz}\right), 8.02\left(1 \mathrm{H},{ }^{3} \mathrm{~J}=8.8 \mathrm{~Hz}\right) \cdot{ }^{13} \mathrm{C}$ NMR $\delta_{\mathrm{c}}$ ppm: 46.5 (1C), $57.5(2 \mathrm{C}), 113.5,115.4,121.6,122.5,123.1$, 123.7, 125.3, 125.5, 126.4, 127.1, 127.6, 127.8, 128.2, 128.4, 128.6, $129.7,129.9,130.1,131.2,132.5,132.6,133.5,133.6,141.9,142.1$, $143.9,148.9$ (integration of the aromatic carbons is ambiguous).

$N^{2}$-(3,5-Dibromobenzyl)-1,1'-binaphthyl-2,2'-diamine (15). Obtained as the third product in the synthesis of compound 13. Eluent: hexanes $/ \mathrm{CH}_{2} \mathrm{Cl}_{2}$ (2:1). Yield $200 \mathrm{mg}$ (16\%). (MALDI-TOF) found: 531.0029. $\mathrm{C}_{27} \mathrm{H}_{21} \mathrm{Br}_{2} \mathrm{~N}_{2}$ requires $531.0072[\mathrm{M}+\mathrm{H}]^{+} .{ }^{1} \mathrm{H} \mathrm{NMR}$ $\delta_{\mathrm{H}}$ ppm: $3.73(2 \mathrm{H}$, br.s), $4.31(2 \mathrm{H}, \mathrm{s}), 4.32(1 \mathrm{H}$, br.s $), 7.03(1 \mathrm{H}, \mathrm{d}$, $\left.{ }^{3} J=9.0 \mathrm{~Hz}\right), 7.11-7.16(2 \mathrm{H}, \mathrm{m}), 7.18\left(1 \mathrm{H}, \mathrm{d},{ }^{3} \mathrm{~J}=8.7 \mathrm{~Hz}\right), 7.20-7.25$ $(2 \mathrm{H}, \mathrm{m}), 7.28-7.34(2 \mathrm{H}, \mathrm{m}), 7.35\left(2 \mathrm{H}, \mathrm{d},{ }^{4} J=1.5 \mathrm{~Hz}\right), 7.51(1 \mathrm{H}, \mathrm{t}$, $\left.{ }^{4} J=1.5 \mathrm{~Hz}\right), 7.78-7.87(4 \mathrm{H}, \mathrm{m}) .{ }^{13} \mathrm{C}$ NMR $\delta_{\mathrm{c}} \mathrm{ppm}: 46.1$ (1C), 111.8 (1C), 112.7 (1C), 113.6 (1C), 118.3 (1C), 122.3 (1C), 122.6 (2C), 123.1 (1C), 123.7 (1C), 123.8 (1C), 126.9 (1C), 127.2 (1C), 127.8 (1C), 128.1 (1C), 128.2 (1C), 128.4 (3C), 129.7 (2C), 132.5 (1C), 133.4 (1C), 133.8 (1C), 142.8 (1C), 142.9 (1C), 144.2 (1C).

Macrocycle 16. A two-neck flask equipped with a magnetic stirrer and reflux condenser is flushed with dry argon, charged with compound 13 (0.25 mmol, $194 \mathrm{mg})$, Pd(dba) ${ }_{2}(16 \mathrm{~mol} \%, 23 \mathrm{mg}$ ), BINAP (18 mol\%, $28 \mathrm{mg})$, absolute dioxane $(12 \mathrm{ml})$, trioxadiamine 2d $(0.5 \mathrm{mmol}, 110 \mathrm{mg})$ is then added followed by sodium tert-butoxide $(0.75 \mathrm{mmol}, 72 \mathrm{mg})$. The reaction mixture is refluxed for $24 \mathrm{~h}$, after cooling it down to ambient temperature the solvent is filtered, the precipitate is washed with $\mathrm{CH}_{2} \mathrm{Cl}_{2}(5 \mathrm{ml})$, combined organic fractions are evaporated in vacuo, and the residue is chromatographed on silica gel using a sequence of eluents: $\mathrm{CH}_{2} \mathrm{Cl}_{2}$, $\mathrm{CH}_{2} \mathrm{Cl}_{2} / \mathrm{MeOH}$ (200:1-50:1). The macrocycle $\mathbf{1 6}$ was obtained with $\mathrm{CH}_{2} \mathrm{Cl}_{2} / \mathrm{MeOH}(100: 1)$ as a yellow viscous oil, yield $28 \mathrm{mg}$ (13\%). (MALDI-TOF) found: 837.1954. $\mathrm{C}_{44} \mathrm{H}_{47} \mathrm{Br}_{2} \mathrm{~N}_{4} \mathrm{O}_{3}$ requires $837.2015[\mathrm{M}+\mathrm{H}]^{+} .{ }^{1} \mathrm{H}$ NMR $\delta_{\mathrm{H}}$ ppm: $1.68\left(4 \mathrm{H}\right.$, quintet, $\left.{ }^{3} J=5.3 \mathrm{~Hz}\right)$, 2.97-3.02 (4H, m), 3.48-3.52 (4H, m), 3.54-3.57 (4H, m), $3.60-3.63(4 \mathrm{H}, \mathrm{m}), 4.19\left(2 \mathrm{H}, \mathrm{dd},{ }^{2} J=15.5 \mathrm{~Hz},{ }^{3} J=4.5 \mathrm{~Hz}\right), 4.27(2 \mathrm{H}$, $\left.\mathrm{dd},{ }^{3} J=5.4 \mathrm{~Hz},{ }^{3} J=4.5 \mathrm{~Hz}\right), 4.34\left(2 \mathrm{H}, \mathrm{dd},{ }^{2} J=15.5 \mathrm{~Hz},{ }^{3} J=5.4 \mathrm{~Hz}\right)$, $6.35(2 \mathrm{H}, \mathrm{s}), 6.53(2 \mathrm{H}, \mathrm{s}), 6.64(2 \mathrm{H}, \mathrm{s}), 7.01-7.05(2 \mathrm{H}, \mathrm{m}), 7.14$ $\left(2 \mathrm{H}, \mathrm{d},{ }^{3} \mathrm{~J}=9.1 \mathrm{~Hz}\right), 7.18-7.23(4 \mathrm{H}, \mathrm{m}), 7.76-7.80(2 \mathrm{H}, \mathrm{m}), 7.83$ $\left(2 \mathrm{H}, \mathrm{d},{ }^{3} J=9.1 \mathrm{~Hz}\right) .{ }^{13} \mathrm{C}$ NMR $\delta_{\mathrm{c}}$ ppm: $28.5(2 \mathrm{C}), 41.7(2 \mathrm{C}), 47.1$ (2C), 69.7 (2C), 70.0 (2C), 70.5 (2C), 109.5 (2C), 111.9 (2C), 113.5 (2C), 114.0 (2C), 117.8 (2C), 122.1 (2C), 123.3 (2C), $123.8(2 \mathrm{C})$, 126.9 (2C), 127.8 (2C), 128.1 (2C), 129.8 (2C), 133.8 (2C), 142.8 (2C), 144.0 (2C), 150.1 (2C).

\section{Results and Discussion}

As BINAM molecule contains two identical primary amino groups, to furnish a macrocyclic structure with poly- amine chain by means of $\operatorname{Pd}(0)$-catalyzed amination, one needs to introduce the aromatic spacer between nitrogen atoms of BINAM and polyamine. At first we compared two approaches for introducing such spacer, the first one employing the reaction of BINAM with $N, N^{\prime}$-bis(bromophenyl) substituted oxadiamine, the second one using the coupling of $N, N$ '-bis(bromophenyl) substituted binaphthalene-2,2'diamine with free oxadiamine. According to the first method, we synthesized $N, N$ '-di(3-bromophenyl) substituted dioxadiamine 2 starting from dioxadiamine 1a according to the known procedure, ${ }^{[17]}$ and then introduced it in the macrocyclization reaction with $(S)$-BINAM using two catalytic systems: $\mathrm{Pd}(\mathrm{dba})_{2} / \mathrm{BINAP}$ and $\mathrm{Pd}(\mathrm{dba})_{2} /$ DavePhos (Scheme 1). The reactions were run in boiling dioxane with equimolar amounts of the reagents $(c=0.02 \mathrm{M})$ in the presence of sodium tert-butoxide taken as a base.

The target macrocycle 3a was obtained by the column chromatography on silica gel and it was found that DavePhos provided slightly better results than BINAP (43\% compared to $34 \%$ ). Here and further all compounds were isolated by column chromatography and reported yields correspond to isolated products. Using the second method, at first $N, N$ '-di(3-bromophenyl) substituted BINAM 4 was synthesized in $68 \%$ yield by reacting BINAM with 2.2 equiv. 1,3-dibromobenzene in the presence of $\mathrm{Pd}(\mathrm{dba})_{2} / \mathrm{Xantphos}$ catalytic system (Scheme 2). The necessity to use Xantphos instead of more universal BINAP ligand is explained by a high reactivity of the amino groups in BINAM that results in the formation of a considerable amount of linear oligomers caused by the diamination of 1,3-dibromobenzene. The second macrocyclization step with dioxadiamine 1a was run using $\mathrm{Pd}(\mathrm{dba})_{2} /$ DavePhos catalytic system and gave the target macrocycle 3a in $53 \%$ yield (Scheme 2) what is a good result for the catalytic macrocyclization reactions. The overall yield of the macrocycle according to the first method was $21 \%$ while in the second method it attained $36 \%$ thus we chose it for the synthesis of other oxadiazamacrocycles. Moreover, the chromatographic isolation of the intermediate compound $\mathbf{4}$ is much easier than that of oxadiamine derivative 2 .

The reactions of other oxadiamines $\mathbf{3 b}$-d differing in the number of the oxygen atoms and chain length were conducted under stated conditions and produced similar results: the yields of the macrocyclization products ranged

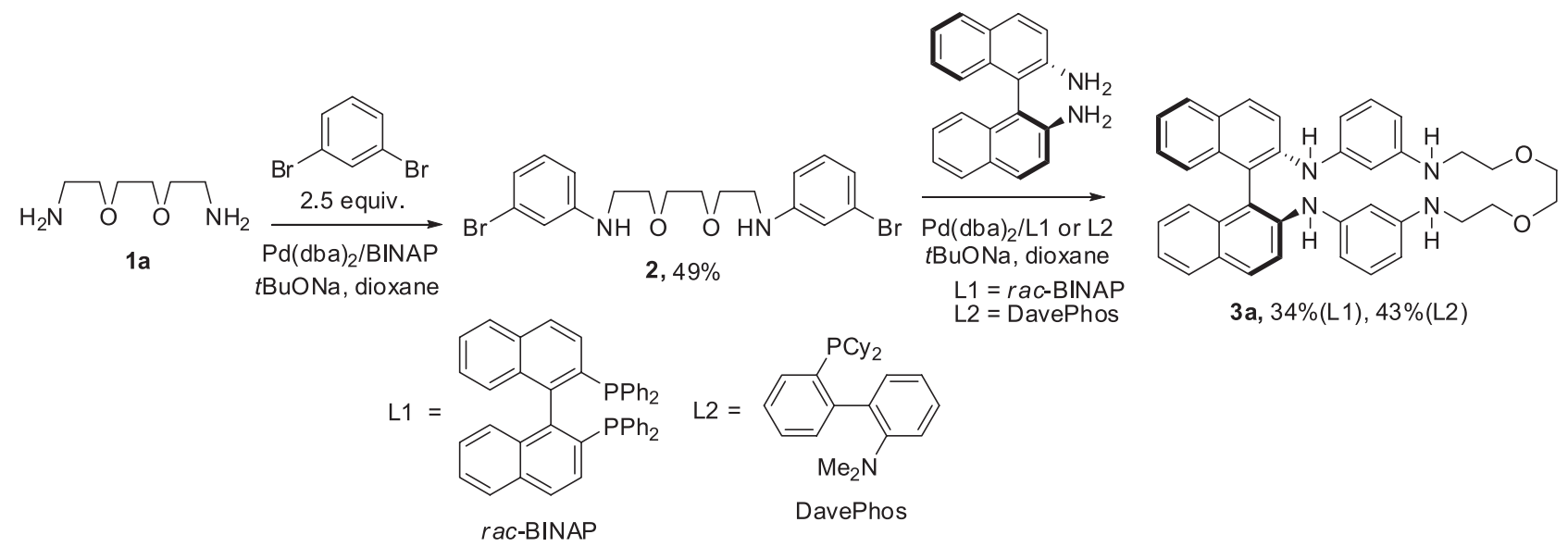

Scheme 1. 


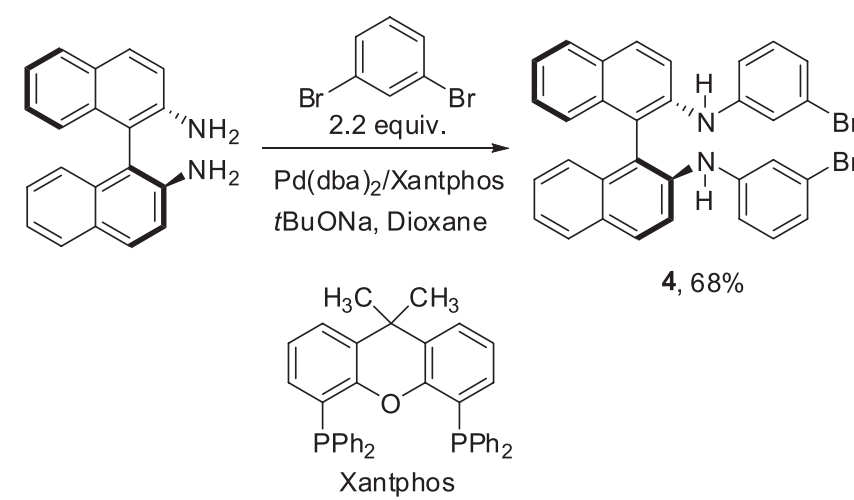

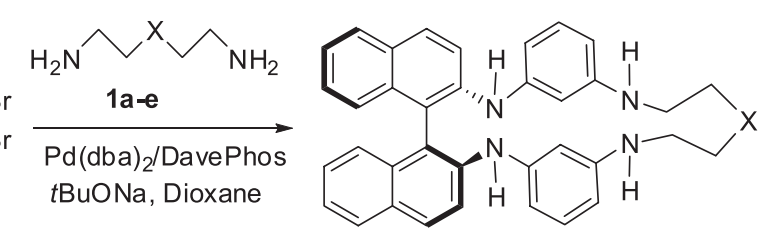

$3 a-e$

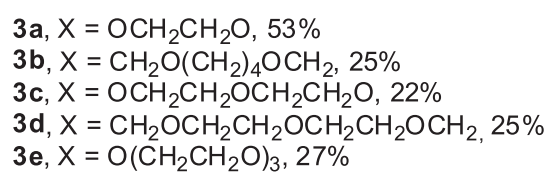

3a, $\mathrm{X}=\mathrm{OCH}_{2} \mathrm{CH}_{2} \mathrm{O}, 53 \%$

$3 \mathrm{~b}, \mathrm{X}=\mathrm{CH}_{2} \mathrm{O}\left(\mathrm{CH}_{2}\right)_{4} \mathrm{OCH}_{2}, 25 \%$

$3 d, X=\mathrm{CH}_{2} \mathrm{OCH}_{2} \mathrm{CH}_{2} \mathrm{OCH}_{2} \mathrm{CH}_{2} \mathrm{OCH}_{2}, 25 \%$

$3 e, X=O\left(\mathrm{CH}_{2} \mathrm{CH}_{2} \mathrm{O}\right)_{3}, 27 \%$

Scheme 2.

from 22 to $27 \%$, thus only the diamine with the shortest chain provided two times higher yield, probably due to a better adjustment of its two amino groups to two bromine atoms in the compound $\mathbf{4}$.

A similar approach was used for the synthesis of the macrocycles possessing two 2,7-disubstituted naphthalene moieties. The reaction of BINAM with 2.5 equiv. of 2,7-dibromonaphthalene afforded $N, N$ '-di(7-bromonaphth-2-yl) derivative 5 in $52 \%$ yield, and this compound was introduced in the series of catalytic macrocyclization reactions with the same oxadiamines 1a-e (Scheme 3). Again the best result was obtained with the shortest oxadiamine 1a (34\% yield of the corresponding macrocycle $6 \mathbf{a}$ ), rather good yield was also provided by another oxadiamine $\mathbf{1 b}$ with a longer chain, (31\% yield of $\mathbf{6 b})$. However, the results with other oxadiamines were less encouraging: trioxadiamine 1d gave $15 \%$ yield of the macrocyclization poroduct $\mathbf{6 d}$, tetraoxadiamine 1e cyclized into the target product only in $7 \%$ yield, while trioxadiamine $\mathbf{6 c}$ provided corresponding macrocycle $\mathbf{6 c}$ only in trace amounts in the reaction mixture. We may propose the same explanation for the observing effect of the chain length as in the case with macrocycles $\mathbf{3}$, but in the reaction of oxadiamines with dinaphthyl derivative $\mathbf{5}$ this dependence is more pronounced due to a longer distance between two bromine atoms participating in the intramolecular cyclization.

In our previous works dealing with the synthesis of macrocycles by $\mathrm{Pd}(0)$-catalyzed intramolecular diamination of dihaloarenes we found out that in many cases the substitution of the second halogen atom proceeded with difficul- ties. $^{[18-21]}$ On the other hand, catalytic macrocyclization of di(bromobenzyl) derivatives afforded enough high yields. ${ }^{[22-25]}$ We attempted the synthesis of $N, N$ '-di(3-bromobenzyl) substituted BINAM by the reaction with 2 equiv. of 3-bromobenzyl bromide in boiling $\mathrm{MeCN}$ in the presence of potassium carbonate and isolated the target compound 7 in $32 \%$ yield (Scheme 4 ). This modest result is explained by the inevitable formation of the side products of tetra- and tribenzylation $\mathbf{8}$ and $\mathbf{9}$, the isomeric $N, N$-di(3-bromobenzyl) derivative 10 and monobenzylated BINAM 11. Compounds 9 and 11 were isolated in pure state (yields 15 and $22 \%$ ), isomer $\mathbf{1 0}$ was obtained and characterized in the mixture with the target compound 7.

Compound 7 was introduced in the macrocyclization reaction with oxadiamines $\mathbf{1 a}, \mathbf{b}, \mathbf{d}$, in this case $\mathrm{Pd}(\mathrm{dba})_{2} /$ BINAP catalytic system was applied as it was observed earlier that BINAP is more helpful for the amination reactions of bromobenzyl derivatives. As it was expected, the macrocyclization reactions proceeded more successfully than in the previous cases, and all target macrocycles $\mathbf{1 2 a}, \mathbf{b}, \mathbf{d}$ were obtained in $41-45 \%$ yields (Scheme 4 ). It is supposed that two factors make the reaction in this case easier: the amination of bromobenzyl moiety proceeds smoother than that of aminobromophenyl moiety, and conformational factors are more favorable in the case of bromobenzyl amination.

At the last step of our investigation we synthesized $N, N$ 'di(3,5-dibromobenzyl) substituted BINAM 13 via a similar reaction with 3,5-dibromobenzyl bromide (Scheme 5), in this process the yield of the target product attained $76 \%$ while
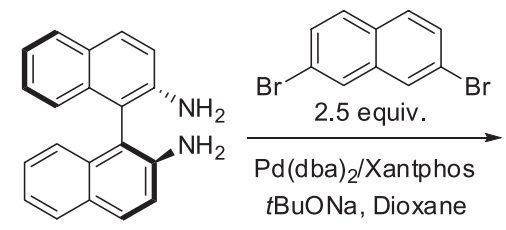
tBuONa, Dioxane

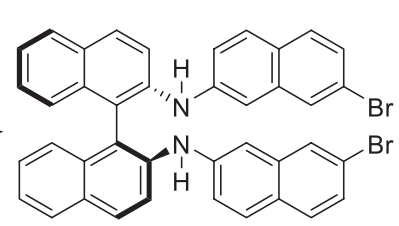

$5,52 \%$

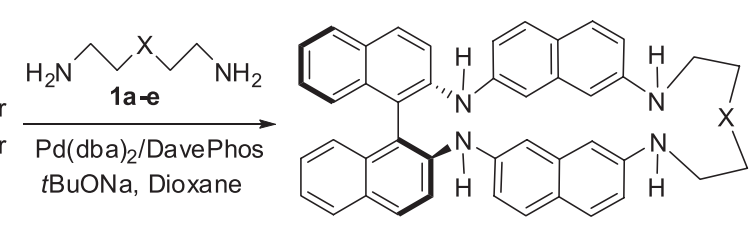

6a, $\mathrm{X}=\mathrm{OCH}_{2} \mathrm{CH}_{2} \mathrm{O}, 34 \%$

6b, $X=\mathrm{CH}_{2} \mathrm{O}\left(\mathrm{CH}_{2}\right)_{4} \mathrm{OCH}_{2}, 31 \%$

6c, $\mathrm{X}=\mathrm{OCH}_{2} \mathrm{CH}_{2} \mathrm{OCH}_{2} \mathrm{CH}_{2} \mathrm{O}$, traces

6d, $\mathrm{X}=\mathrm{CH}_{2} \mathrm{OCH}_{2} \mathrm{CH}_{2} \mathrm{OCH}_{2} \mathrm{CH}_{2} \mathrm{OCH}_{2}, 15 \%$

6e, $\mathrm{X}=\mathrm{O}\left(\mathrm{CH}_{2} \mathrm{CH}_{2} \mathrm{O}\right)_{3}, 7 \%$ 


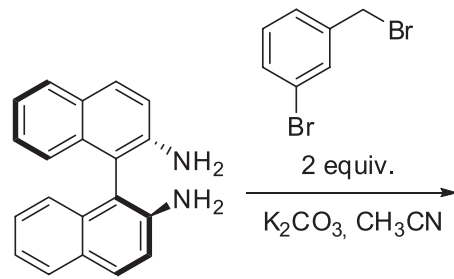<smiles>Brc1cccc(CNc2ccc3ccccc3c2C2=c3ccccc3=CC[C@H]2NCc2cccc(Br)c2)c1</smiles>

7, $32 \%$

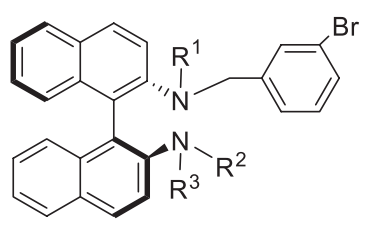

8: $R^{1}=R^{2}=R^{3}=3-B C_{6} \mathrm{H}_{4}$, traces 9: $\mathrm{R}^{1}=\mathrm{H}, \mathrm{R}^{2}=\mathrm{R}^{3}=3-\mathrm{BrC}_{6} \mathrm{H}_{4}, 15 \%$

10: $\mathrm{R}^{1}=3-\mathrm{BrC}_{6} \mathrm{H}_{4}, \mathrm{R}^{2}=\mathrm{R}^{3}=\mathrm{H}, 5 \%$

11: $R^{1}=R^{2}=R^{3}=H, 22 \%$ (in mixture with 7)<smiles>Brc1cccc(CN2Cc3ccc4ccccc4c3C3=C4C=Cc5ccccc5C=C3NCN4C2)c1</smiles><smiles>[X]CCCNc1cccc(CNc2cccc3ccccc23)c1NCc1cccc2ccccc12</smiles>

12a, $\mathrm{X}=\mathrm{OCH}_{2} \mathrm{CH}_{2} \mathrm{O}, 41 \%$

12b, $\mathrm{X}=\mathrm{CH}_{2} \mathrm{O}\left(\mathrm{CH}_{2}\right)_{4} \mathrm{OCH}_{2}, 44 \%$

12d, $X=\mathrm{CH}_{2} \mathrm{OCH}_{2} \mathrm{CH}_{2} \mathrm{OCH}_{2} \mathrm{CH}_{2} \mathrm{OCH}_{2}, 45 \%$

Scheme 4.<smiles>[X]C([R])=O</smiles><smiles>CN(Cc1cc(Br)cc(Br)c1)c1ccc2ccccc2c1-c1c(NCc2cc(Br)cc(Br)c2)ccc2ccccc12</smiles><smiles>[R]Nc1ccc2ccccc2c1-c1c(N(Cc2cc(Br)cc(Br)c2)Cc2ccc3ccccc3c2)ccc2ccccc12</smiles>

14: $R^{1}=R^{2}=3,5-d_{i B r C} \mathrm{H}_{3}, 9 \%$ 15: $R^{1}=R^{2}=H, 16 \%$<smiles>CN(Cc1cc(Br)cc(Br)c1)c1ccc2ccccc2c1-c1c(N(Cc2cc(Br)cc(Br)c2)Cc2cc(Br)cc(Br)c2)ccc2ccccc12</smiles>

13<smiles>NCCCOCCOCCOCCCN</smiles>

1d, 2 equiv.

$\mathrm{Pd}(\mathrm{dba})_{2} / \mathrm{BINAP}$ $t$ BuONa, dioxane

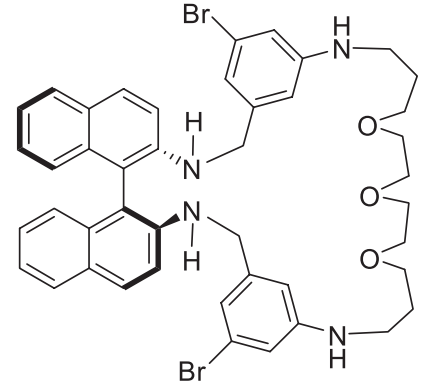

$16,13 \%$

Scheme 5 .

tribenzylated and monobenzylated by-products $\mathbf{1 4}$ and $\mathbf{1 5}$ were isolated in 9 and $16 \%$, respectively. The compound $\mathbf{1 3}$ was introduced in the reaction with 2 equiv. of trioxadiamine 1d using $\mathrm{Pd}(\mathrm{dba})_{2} / \mathrm{BINAP}$ catalytic system in view of the synthesis of macrobicyclic compound, however, only macrocycle 16 was obtained in $13 \%$ yield. The substitution of one bromine atom in each benzyl substituent took place and no evidence of the diamination of the phenyl ring could be received from the ${ }^{1} \mathrm{H}$ NMR spectrum of the reaction mixture. We tried another donor phosphine ligand, RuPhos which was thought to promote diamination, and in the reaction mixture weak signals of 3,5-diaminobenzyl group were observed in ${ }^{1} \mathrm{H}$ NMR spectrum, but no product could be isolated by chromatography in this case. 


\section{Conclusions}

To sum up, we developed the catalytic approach to a previously unknown class of $\mathrm{N}$ - and $\mathrm{O}$-containing macrocycles comprising chiral BINAM fragment. The method consists of two subsequent $\mathrm{Pd}(0)$-catalyzed amination reactions - the first step is the synthesis of corresponding $N, N^{\prime}$ di(bromoaryl) substituted BINAM, the second is $\operatorname{Pd}(0)$ mediated macrocyclization with linear oxadiamines. This approach was demonstrated to be enough general for the synthesis of macrocycles possessing various aryl spacers (phenyl, benzyl and naphthalene) and oxadiamine chains. The dependence of the compounds yields on the structure of starting compounds was established. The reaction of $N, N$ 'di(3,5-dibromobenzyl) substituted BINAM with trioxadiamine led to substitution of one bromine atom in each benzyl moiety and formation of a similar macrocyclic compound.

Acknowledgements. This work was financially supported by the RFBR grant 15-03-04698.

\section{References}

1. Piaztek P., Kalisiak J., Jurczak J. Tetrahedron Lett. 2004, 45, 3309-3311.

2. Toyota S. Chem. Lett. 2011, 40, 12-18.

3. Ogoshi T., Masaki K., Shiga R., Kitajima K., Yamagishi T. Org. Lett. 2011, 13, 1264-1266.

4. Ranyuk E.R., Averin A.D., Beletskaya I.P. Adv. Synth. Catal. 2010, 352, 2299-2305.

5. Kobelev S.M., Averin A.D., Maloshitskaya O.A., Denat F., Guilard R., Beletskaya I.P. Macroheterocycles 2012, 5, 389395.

6. Averin A.D., Ranyuk E.R., Lukashev N.V., Beletskaya I.P. Chem. Eur. J. 2005, 11, 1730-1739.

7. Averin A.D., Ranyuk E.R., Lukashev N.V., Golub S.L., Buryak A.K., Beletskaya I.P. Tetrahedron Lett. 2008, 49, 1188-1191.
8. Lukashev N.V., Kazantsev A.V., Averin A.D., Donez P.A., Baranov M.S., Sievanen E., Kolehmainen E. Synthesis 2009, 2009, 4175-4182.

9. Latyshev G.V., Baranov M.S., Kazantsev A.V., Averin A.D., Lukashev N.V., Beletskaya I.P. Synthesis 2009, 2009, 26052612.

10. Izatt R.M., Pawlak K., Bradshaw J.S. Chem. Rev. 1991, 91 , 1721-2085.

11. Pu L. Acc. Chem. Res. 2012, 45, 150-163.

12. Yu S., Pu L. Science China 2013, 56, 301-306.

13. Li Z.-B., Lin J., Zhang H.-C., Sabat M., Hyacinth M., Pu L. J. Org. Chem. 2004, 69, 6284-6289.

14. Li Z.-B., Lin J., Sabat M., Hyacinth M., Pu L. J. Org. Chem. 2007, 72, 4905-4916.

15. Ukai T., Kawazura H., Ishii Y., Bonnet J.J., Ibers J.A. J. Organomet. Chem. 1974, 65, 253-266.

16. Neenan T.X., Whitesides G.M. J. Org. Chem. 1988, 53, 24892496.

17. Averin A.D., Shukhaev A.V., Golub S.L., Buryak A.K., Beletskaya I.P. Synthesis 2007, 2995-3012.

18. Beletskaya I.P., Averin A.D., Pleshkova N.A., Borisenko A.A., Serebryakova M.V., Denat F., Guilard R. Synlett 2005, 87-90.

19. Averin A.D., Ulanovskaya O.A., Borisenko A.A., Serebryakova M.V., Beletskaya I.P. Tetrahedron Lett. 2006, 47, 26912694.

20. Averin A.D., Uglov A.N., Beletskaya I.P. Chem. Lett. 2008, 37, 1074-1075.

21. Abel A.S., Averin A.D., Beletskaya I.P. New J. Chem. 2016, 40, 5818-5828.

22. Kobelev S.M., Averin A.D., Buryak A.K., Vovk A.I., Kukhar V.P., Denat F., Guilard R., Beletskaya I.P. Heterocycles 2015, 90, 989-1017.

23. Averin A.D., Shukhaev A.V., Vovk A.I., Kukhar V.P., Denat F., Guilard R., Beletskaya I.P. Macroheterocycles 2014, 7, 174180.

24. Yakushev A.A., Chernichenko N.M., Anokhin M.V., Averin A.D., Buryak A.K., Denat F., Beletskaya I.P. Molecules 2014, 19, 940-965.

25. Uglov A.N., Zubrienko G.A., Abel A.S., Averin A.D., Maloshitskaya O.A., Bessmertnykh-Lemeune A., Denat F., Beletskaya I.P. Heterocycles 2014, 88, 1213-1231. 